DOI 10.4171/JEMS/239

L. Berlyand · V. Rybalko

\title{
Solutions with vortices of a semi-stiff boundary value problem for the Ginzburg-Landau equation
}

Received February 15, 2008 and in revised form October 17, 2008

Abstract. We study solutions of the 2D Ginzburg-Landau equation

$$
-\Delta u+\frac{1}{\varepsilon^{2}} u\left(|u|^{2}-1\right)=0
$$

subject to "semi-stiff" boundary conditions: Dirichlet conditions for the modulus, $|u|=1$, and homogeneous Neumann conditions for the phase. The principal result of this work shows that there are stable solutions of this problem with zeros (vortices), which are located near the boundary and have bounded energy in the limit of small $\varepsilon$. For the Dirichlet boundary condition ("stiff" problem), the existence of stable solutions with vortices, whose energy blows up as $\varepsilon \rightarrow 0$, is well known. By contrast, stable solutions with vortices are not established in the case of the homogeneous Neumann ("soft") boundary condition.

In this work, we develop a variational method which allows one to construct local minimizers of the corresponding Ginzburg-Landau energy functional. We introduce an approximate bulk degree as the key ingredient of this method; unlike the standard degree over the curve, it is preserved in the weak $H^{1}$-limit.

\section{Introduction and main results}

In this work, we study solutions of the Ginzburg-Landau (GL) equation

$$
-\Delta u+\frac{1}{\varepsilon^{2}} u\left(|u|^{2}-1\right)=0 \quad \text { in } A,
$$

where $\varepsilon$ is a positive parameter (the inverse of the GL parameter $\kappa=1 / \varepsilon$ ), $u$ is a complexvalued ( $\mathbb{R}^{2}$-valued) map, and $A$ is a smooth, bounded, multiply connected domain in $\mathbb{R}^{2}$. For simplicity, hereafter we assume $A$ is an annular type (doubly connected) domain of the form $A=\Omega \backslash \bar{\omega}$, where $\Omega$ and $\omega$ are simply connected smooth domains and $\bar{\omega} \subset \Omega \subset \mathbb{R}^{2}$.

Equation $(1.1)$ is the Euler-Lagrange PDE corresponding to the energy functional

$$
E_{\varepsilon}(u)=\frac{1}{2} \int_{A}|\nabla u|^{2} d x+\frac{1}{4 \varepsilon^{2}} \int_{A}\left(|u|^{2}-1\right)^{2} d x .
$$

L. Berlyand: Department of Mathematics, The Pennsylvania State University, University Park, PA 16802, USA; e-mail: berlyand@math.psu.edu

V. Rybalko: Mathematical Division, Institute for Low Temperature Physics and Engineering, 47 Lenin Ave., 61103 Kharkov, Ukraine; e-mail: vrybalko@ilt.kharkov.ua 
Equations of this type arise, e.g., in models of superconductivity and superfluidity. Additionally, (1.1) is viewed as a complex-valued version of the Allen-Cahn model for phase transitions [31].

Solutions of (1.1) subject to Dirichlet boundary conditions, $u=g$ on $\partial A$ with fixed $S^{1}$-valued boundary data $g$, have been extensively studied in the past decade. Special attention has been paid to solutions with isolated zeros (vortices). In contrast with the Dirichlet problem, in the case of the homogeneous Neumann boundary condition, solutions are typically vortexless; in particular, stable solutions with vortices have not been found.

This work is devoted to finding the solutions of 1.1 subject to the "semi-stiff" boundary conditions

$$
|u|=1 \quad \text { and } \quad u \times \frac{\partial u}{\partial v}=0 \quad \text { on } \partial A
$$

These boundary conditions are intermediate between Dirichlet and Neumann in the following sense: any solution $u \in H^{1}\left(A ; \mathbb{R}^{2}\right)$ of $1.1,1.3$ is sufficiently regular [7], so it can be written as $u=|u| e^{i \psi}$ (locally) near the boundary. Then 1.3 means that Dirichlet boundary conditions are prescribed for modulus, $|u|=1$ on $\partial A$, and Neumann conditions are prescribed for the phase, $\partial \phi / \partial v=0$ on $\partial A$.

Problem 1.1 1.3 is equivalent to finding critical points of the energy functional 1.2 in the space

$$
\mathcal{J}=\left\{u \in H^{1}\left(A ; \mathbb{R}^{2}\right) ;|u|=1 \text { a.e. on } \partial A\right\} .
$$

Our main objective is to study the existence of stable solutions of $(1.1,1.3)$ with vortices. Since the problem is time independent, stable solutions are defined as (local) minimizers of $(1.2)$ in $\mathcal{J}$. In other words, we are interested in whether the model (1.1, 1.3) stabilizes vortices similarly to the Dirichlet problem or does not stabilize vortices analogously to the Neumann problem. The boundary conditions (1.3) are not well studied, and this work, along with studies [10, 19, 7, 8, 9], reveals their distinctive features, described later in the Introduction.

Let us briefly review the existing results for the Dirichlet and Neumann boundary value problems for equation (1.1). The first results on the existence of stable solutions with vortices for the Dirichlet problem were obtained in [17, 18]. Stable solutions of (1.1) with vortices were obtained and studied in [11] for star-shaped domains and prescribed $S^{1}$-valued boundary data with nonzero topological degree. In [11], the limiting locations (as $\varepsilon \rightarrow 0$ ) of the vortices of the global minimizers and other solutions with vortices (if they exist) are described by means of a renormalized energy. Subsequently, these results were generalized to multiply connected domains in [33]. The existence of locally minimizing and minmax solutions was established first in [23] and [24], then in more detail and generality in [27] (see also [4, 15]). We refer the reader to [13], and references therein, for the various results on the Dirichlet problem. As mentioned above, only vortexless stable solutions of (1.1) with the homogeneous Neumann boundary condition in 2D are known. Moreover, all locally minimizing solutions are constant maps if $A$ is convex [20], or simply connected and $\varepsilon$ is small [32]. The existence of nonconstant (but vortexless) locally minimizing solutions is established in [21] and [3]. In the recent work [15], 
a general result for the existence of (nonminimizing) solutions with vortices have been found. Similarly to the Dirichlet problem, these solutions with vortices have energy that blows up as $\varepsilon \rightarrow 0$.

Equation (1.1) (and functional (1.2) is usually referred to as a simplified GL model (without magnetic field). There is a large body of mathematical literature on the general GL model with a magnetic field (e.g., [29, 5, 2, 28, 22]). Since (1.1) is obtained from the general GL energy by setting the magnetic field equal to zero, it describes the persistent currents in a 2D cross-section of a cylindrical superconductor (or in a 2D film). It was observed in [11] that the degree of the boundary data on connected components of $\partial A$ creates the same type of "quantized vortices" as a magnetic field in type II superconductors or as angular rotation in superfluids. Despite a relatively simple form of equation (1.1), it leads to a deep analysis of properties of its solutions similar to other fundamental PDE's in mathematical physics.

The boundary conditions 1.3 model, e.g., the surface of a superconductor coated with a high temperature superconducting thin film. Generating a mathematical model of persistent currents in such a superconductor then amounts to finding critical points of functional 1.2$\}$ in the space $\mathcal{J}$, when $u=|u| e^{i \psi}$ on the boundary and $|u|=1$, while the phase $\psi$ is "free".

Boundary conditions $[1.3$ appeared in recent studies [10, 19, 8] of the minimization problem for the GL functional (1.2) among maps from $\mathcal{J}$ with prescribed degrees on the connected components of the boundary. The minimization of the energy $(1.2)$ in $\mathcal{J}$ produces only constant solutions of $(1.1,1.3)$, similar to the case of the Neumann problem, which corresponds to finding critical points of $[1.2)$ in the entire space $H^{1}\left(A ; \mathbb{R}^{2}\right)$. An obvious way of producing critical points with vortices is to impose two different degrees $q \neq p$ on $\partial \Omega$ and $\partial \omega$; that is, to consider the minimization of $E_{\varepsilon}(u)$ in the set $\mathcal{J}_{p q} \subset \mathcal{J}$, where

$$
\mathcal{J}_{p q}:=\{u \in \mathcal{J} ; \operatorname{deg}(u, \partial \omega)=p, \operatorname{deg}(u, \partial \Omega)=q\} .
$$

Recall that the degree (winding number) of a map $u \in H^{1 / 2}\left(\gamma ; S^{1}\right)$ on $\gamma$ (where $\gamma$ is either $\partial \omega$ or $\partial \Omega$ ) is an integer given by the classical formula (cf., e.g., [12])

$$
\operatorname{deg}(u, \gamma)=\frac{1}{2 \pi} \int_{\gamma} u \times \frac{\partial u}{\partial \tau} d \tau
$$

where the integral is understood via $H^{1 / 2}-H^{-1 / 2}$ duality, and $\partial / \partial \tau$ is the tangential derivative with respect to the counterclockwise orientation of $\gamma$. (Throughout the paper we assume the same orientation of $\partial \omega$ and $\partial \Omega$.) Note that $\mathcal{J}_{p q}$ are connected components of $\mathcal{J}$ (see [12]).

Simple topological considerations imply that critical points from $\mathcal{J}_{p q}$ must have at least $|p-q|$ vortices (with multiplicity). We emphasize that the existence of such critical points is far from obvious. For example (see Section 2), there are no global minimizers of $E_{\varepsilon}(u)$ in $\mathcal{J}_{01}$ and the weak limits of minimizing sequences do not belong to $\mathcal{J}_{01}$. This simple example illustrates an important property of the sets $\mathcal{J}_{p q}$, which is crucial for our considerations: these sets are not weakly $H^{1}$-closed, since the degree at the boundary may change in the weak $H^{1}$-limit. Thus the direct methods of the calculus of variations cannot 
be used. On the other hand, the results of this work show that when $p=q$ and there is no topological reason for vortices to appear, local minimizers typically do have vortices.

As mentioned above, the vortex structure of solutions of (1.1) with Dirichlet and Neumann boundary conditions is well studied. In contrast, only vortexless solutions of the semi-stiff problem [1.1] 1.3) have been found [8, 19]. In [8], it was shown that minimizing sequences for the corresponding minimization problem develop a novel type of so-called "near-boundary" vortices, which approach the boundary and have finite GL energy in the limit of small $\varepsilon$. However, such minimizing sequences do not converge to actual minimizers [9]. These studies lead to the natural question of whether there exist true solutions of 1.1. 1.3 with near-boundary vortices. Unlike the minimizing sequences, such solutions may model observable states of a physical system (e.g., persistent currents with vortices and superfluids between rotating cylinders [16]). The following theorem, which is the main result of this work, provides an answer to this question.

Theorem 1 (Existence of solutions with vortices of problem (1.1, 1.3)). For any integer $M>0$, there exist at least $M$ distinct stable solutions of (1.1. 1.3 with (near-boundary) vortices when $\varepsilon<\varepsilon_{1}\left(\varepsilon_{1}=\varepsilon_{1}(M)>0\right)$. The vortices of these solutions are at distance $o(\varepsilon)$ from the boundary and have bounded GL energy in the limit $\varepsilon \rightarrow 0$. The solutions are stable in the sense that they are (local) minimizers of (1.2) in $\mathcal{J}$.

To construct local minimizers of (1.2) in $\mathcal{J}$, we represent $\mathcal{J}$ as the union of subsets $\mathcal{J}_{p q}^{(d)}$ (defined in 1.8 below), $\mathcal{J}=\bigcup_{p, q, d \in \mathbb{Z}} \mathcal{J}_{p q}^{(d)}$, and study the existence of global minimizers in $\mathcal{J}_{p q}^{(d)}$. Furthermore, we show that each minimizer lies in $\mathcal{J}_{p q}^{(d)}$ with its open neighborhood. Therefore, the minimizers in $\mathcal{J}_{p q}^{(d)}$ are distinct local minimizers in $\mathcal{J}$.

Thus, the construction of solutions of (1.1, 1.3) is based on the study of the following constrained minimization problem:

$$
m_{\varepsilon}(p, q, d):=\inf \left\{E_{\varepsilon}(u) ; u \in \mathcal{J}_{p q}^{(d)}\right\}
$$

where

$$
\mathcal{J}_{p q}^{(d)}=\left\{u \in \mathcal{J}_{p q} ; d-1 / 2 \leq \operatorname{abdeg}(u) \leq d+1 / 2\right\},
$$

$p, q$ and $d$ are given integers, and abdeg $(u)$ is the approximate bulk degree, introduced as follows. Consider the boundary value problem

$$
\left\{\begin{array}{l}
\Delta V=0 \quad \text { in } A, \\
V=1 \quad \text { on } \partial \Omega, \\
V=0 \quad \text { on } \partial \omega .
\end{array}\right.
$$

Introduce abdeg $(\cdot): H^{1}\left(A ; \mathbb{R}^{2}\right) \rightarrow \mathbb{R}$ by the formula

$$
\operatorname{abdeg}(u)=\frac{1}{2 \pi} \int_{A} u \times\left(\partial_{x_{1}} V \partial_{x_{2}} u-\partial_{x_{2}} V \partial_{x_{1}} u\right) d x,
$$


where $V$ solves (1.9). In the particular case where $A$ is a circular annulus, $A_{R_{1} R_{2}}=$ $\left\{x ; R_{1}<|x|<R_{2}\right\}, \operatorname{abdeg}(u)$ is expressed by

$$
\operatorname{abdeg}(u)=\frac{1}{\log \left(R_{2} / R_{1}\right)} \int_{R_{1}}^{R_{2}}\left(\frac{1}{2 \pi} \int_{|x|=\xi} u \times \frac{\partial u}{\partial \tau} d s\right) \frac{d \xi}{\xi} .
$$

For $S^{1}$-valued maps, abdeg $(u)$ becomes integer valued and the representation 1.11 clarifies its interpretation as an average value of the standard degree. The definition (1.10) is motivated by the following intuitive consideration: represent the standard degree over the boundary $\partial \Omega$ via a "bulk" integral over the area of $A$ for $S^{1}$-valued maps and notice that if $E_{\varepsilon}(u) \leq \Lambda$ for some finite $\Lambda$ and sufficiently small $\varepsilon$, then $u$ is "almost" $S^{1}$-valued.

It was observed in [3] that for $S^{1}$-valued maps in an annulus $A$, one can define the topological degree $\operatorname{deg}(u, A)$ that classifies maps $u \in H^{1}\left(A ; S^{1}\right)$ according to their 1homotopy type [34] (1-homotopy type is completely determined by the degree of the restriction to a nontrivial contour). This definition was extended in [3] to maps that are not necessarily $S^{1}$-valued by considering $u /|u|$ in a subdomain $A_{u} \subset A$, which is obtained by removing neighborhoods of the boundary $\partial A$ and zeros (vortices) of $u$. Definition (1.11) does not require the removal of vortices from $A$, and $\operatorname{abdeg}(u)$ is obtained by a simple formula (unlike $\operatorname{deg}(u, A)$ in [3], where the domain of integration depends on $u$ ). Note that in general abdeg $(u)$ is not an integer. The most important fact for our considerations is that $\operatorname{abdeg}(u)$ is continuous with respect to weak $H^{1}$-convergence, unlike the standard degree in (1.6) (this issue for $\operatorname{deg}(u, A)$ was not addressed in [3]).

The minimization in problem 1.7 is taken over $\mathcal{J}_{p q}^{(d)}$, which is not an open set, and therefore minimizers of (1.7) (if they exist) are not necessarily local minimizers of (1.2) in $\mathcal{J}$. Indeed, while $\mathcal{J}_{p q}$ is an open subset of $\mathcal{J}$ (hereafter we assume the topology and convergence in $\mathcal{J}$ to be the strong $H^{1}$ unless otherwise specified), the constraint $\operatorname{abdeg}(u) \in[d-1 / 2, d+1 / 2]$ defines a closed set with respect to both strong and weak $H^{1}$-convergences. However, if we further consider a subset of maps with bounded energy and choose $\varepsilon$ small enough, then the constraint abdeg $(u) \in[d-1 / 2, d+1 / 2]$ becomes open due to the following proposition:

Proposition 2. Fix $\Lambda>0$. There exists $\varepsilon_{0}=\varepsilon_{0}(\Lambda)>0$ such that if $0<\varepsilon<\varepsilon_{0}$, then for any integer $d$ and any $u \in H^{1}\left(A ; \mathbb{R}^{2}\right)$ satisfying $E_{\varepsilon}(u) \leq \Lambda$ the closed constraint $\operatorname{abdeg}(u) \in[d-1 / 2, d+1 / 2]$ is equivalent to an open one, that is,

$$
d-1 / 2 \leq \operatorname{abdeg}(u) \leq d+1 / 2 \Leftrightarrow d-1 / 2<\operatorname{abdeg}(u)<d+1 / 2 .
$$

(Actually, it will be shown that $\operatorname{abdeg}(u)$ is close to integers uniformly in $u$ satisfying $E_{\varepsilon}(u) \leq \Lambda$ when $\varepsilon$ is sufficiently small.) The following theorem is the main tool in proving the existence of local minimizers.

Theorem 3 (Existence of minimizers of the constrained problem). For any integers $p, q$ and $d>0[d<0]$ with $d \geq \max \{p, q\}[d \leq \min \{p, q\}]$ there exists $\varepsilon_{1}=\varepsilon_{1}(p, q, d)>0$ such that the infimum in (1.7) is always attained when $\varepsilon<\varepsilon_{1}$. Moreover

$$
m_{\varepsilon}(p, q, d) \leq I_{0}(d, A)+\pi(|d-p|+|d-q|),
$$


where

$$
I_{0}(d, A)=\min \left\{\frac{1}{2} \int_{A}|\nabla u|^{2} d x ; u \in H^{1}\left(A ; S^{1}\right) \cap \mathcal{J}_{d d}\right\} .
$$

The value $I_{0}(d, A)$ is expressed by $I_{0}(d, A)=2(\pi d)^{2} / \operatorname{cap}(A)$ via the $H^{1}$-capacity $\operatorname{cap}(A)$ of the domain $A$.

The key difficulty is to establish the attainability of the infimum in (1.7), which is highly nontrivial since the degree on $\partial \Omega$ and $\partial \omega$ is not preserved in the weak $H^{1}$-limit [8, 9]. We show that the solutions of (1.1, 1.3), which are the minimizers of (1.7) (local minimizers of $[1.2$ in $\mathcal{J}$ ) with $p \neq d$ and any $q$ (or $q \neq d$ and any $p$ ) must have vortices. For fixed $\varepsilon$, these vortices are located at a positive distance from $\partial A$ and approach $\partial A$ as $\varepsilon \rightarrow 0$.

Without loss of generality, throughout this work we always assume that $d>0$ (otherwise one can reverse the orientation of $\mathbb{R}^{2}$ ).

Theorem 1 follows from Theorem 3 and Proposition 2 . The asymptotic behavior of the local minimizers is established in

Theorem 4 (Asymptotic behavior of minimizers and their energies). Assume that the integers $p, q$ and $d$ satisfy the assumptions of Theorem 3. Then as $\varepsilon \rightarrow 0$ the minimizers $u_{\varepsilon}$ of 1.7 converge weakly in $H^{1}(A)$, up to a subsequence, to a harmonic map $u$ which minimizes (1.14). Additionally,

$$
\begin{aligned}
& E_{\varepsilon}\left(u_{\varepsilon}\right)=I_{0}(d, A)+\pi(|d-p|+|d-q|)+o(1) \quad \text { as } \varepsilon \rightarrow 0, \\
& E_{\varepsilon}\left(u_{\varepsilon}\right)=\frac{1}{2} \int_{A}\left|\nabla u_{\varepsilon}\right|^{2} d x+o(1) \quad \text { as } \varepsilon \rightarrow 0 .
\end{aligned}
$$

In particular, it follows from $1.15,1.16$ that there is no strong convergence of the minimizers of 1.7 in $H^{1}(A)$ as $\varepsilon \rightarrow 0$ unless $\pi(|d-p|+|d-q|)=0$. For $p=q=d=1$, the minimizers of problem 1.7 actually coincide with local minimizers of 1.2$)$ in $\mathcal{J}_{11}$ established in [7]. While work [8] is primarily concerned with global minimizers in $\mathcal{J}_{11}$, in its preprint version [7] the issue of local minimizers was raised. Namely it was observed that the vortexless local minimizers of $(1.2)$ in $\mathcal{J}_{11}$ can be obtained by adapting the method of [28], that is, by seeking local minimizers that are $H^{1}$-close as $\varepsilon \rightarrow 0$ to those of problem 1.14 for $S^{1}$-valued maps. This method, however, does not allow one to find local minimizers with vortices.

The techniques of finding local minimizers and other critical points with vortices for the GL functional (with and without magnetic field) are developed, e.g., in [4, 15, 23, 24, 27, 30]. These works establish solutions with vortices distanced from the boundary (inner vortices) and use the reduction to an appropriate renormalized energy function of finitely many variables. For instance, in [30] local minimizers with $n$ vortices are obtained for the full GL energy functional by restricting the minimization to some open sets of the type $U_{n}=\left\{(u, A) ; \pi(n-1)|\log \varepsilon|<G_{\varepsilon}^{0}(u, A)<\pi(n+1)|\log \varepsilon|\right\}$, where $G_{\varepsilon}^{0}(u, A)$ is the energy without external magnetic field. Then the "hard" part of the analysis is to show that the infimum is attained inside $U_{n}$. Similarly, the main difficulty in establishing local minimizers of $(1.2)$ in $\mathcal{J}$ is to show the attainability of the infimum in (1.7). Note that in 1.7$)$ we impose constraints on the approximate bulk degree abdeg $(u)$ rather than 
the energy constraints and also prescribe degrees on the connected components of the boundary.

The method proposed in our work does not use the renormalized energy. Indeed, the asymptotic expansion of energy [1.15) (at least to the leading order as it is used for the inner vortices) does not provide enough information on the location of vortices that approach the boundary. The same difficulty was observed in [8] in the study of the existence/nonexistence of minimizers. We avoid the hard problem of construction of next order renormalized energy (or equivalently next order in the expansion $(1.15)$ ) by a direct construction of test functions with near-boundary vortices that approximate actual minimizers. Our approach to problem (1.7) involves such tools as the Price Lemma [8] and its "uniform" version (see Lemma $[18$ together with tight upper bounds explicitly constructed for fixed (sufficiently small) $\varepsilon$.

Next, we summarize the distinctive features of the GL boundary value problem with semi-stiff boundary conditions. The first interesting feature is the existence of solutions with a new type of vortices called near-boundary vortices. Unlike the inner vortices, whose energy blows up at the rate of $|\log \varepsilon|$, the energy of near-boundary vortices is bounded as $\varepsilon \rightarrow 0$ and they are located at a distance $o(\varepsilon)$ from the boundary.

Secondly, the semi-stiff boundary conditions result in a lack of compactness. Namely, as of now, the only way to find nonconstant minimizers is by searching for minimizers in subsets $\mathcal{J}_{p q}^{(d)} \subset \mathcal{J}$. These subsets, however, are not weakly $H^{1}$-closed and therefore a weak $H^{1}$-limit of a minimizing sequence $\left\{u^{(k)}\right\} \in \mathcal{J}_{p q}^{(d)}$ may not lie in $\mathcal{J}_{p q}^{(d)}$, but rather in $\mathcal{J}_{p^{\prime} q^{\prime}}^{(d)}$ with $p^{\prime} \neq p$ or (and) $q^{\prime} \neq p$. Theorem 3 shows that if $d>0$ and $d \geq \max \{p, q\}$, then (for small $\varepsilon$ ) any weak $H^{1}$-limit of a minimizing sequence $\left\{u^{(k)}\right\} \subset \mathcal{J}_{p q}^{(d)}$ always belongs to $\mathcal{J}_{p q}^{(d)}$, despite the lack of weak $H^{1}$-closedness of $\mathcal{J}_{p q}^{(d)}$. In contrast, if $d \geq 0$ and $d<\max \{p, q\}$ we have

Conjecture 5. Let $d \geq 0, d<\max \{p, q\}$ (or $d \leq 0, d>\min \{p, q\})$ and let $u$ be a weak limit of a minimizing sequence for problem (1.7) (such a minimizing sequence exists and bound 1.13 holds for any integer $p, q, d)$. Then $u \notin \mathcal{J}_{p q}^{(d)}$ when $\varepsilon$ is sufficiently small.

In the simplest case, when $d=0$ and either $p=1$ and $q=0$ or $p=0$ and $q=1$, this conjecture is demonstrated by an argument quite similar to the nonexistence proof in [7] for simply connected domains (see Sec. 2 below).

A more interesting example, which supports the conjecture above, follows from the previously studied (global) minimization problem $\tilde{m}_{\varepsilon}=\inf \left\{E_{\varepsilon}(u) ; u \in \mathcal{J}_{11}\right\}$. It was shown in [7, 9] that if $\operatorname{cap}(A) \geq \pi$ (subcritical/critical cases), then $\tilde{m}_{\varepsilon}$ is always attained, whereas if $\operatorname{cap}(A)<\pi$ (supercritical case), then $\tilde{m}_{\varepsilon}$ is never attained for small $\varepsilon$. One can see that the elements of minimizing sequences lie in $\mathcal{J}_{11}^{(1)}$ in subcritical/critical cases and in $\mathcal{J}_{11}^{(0)}$ in the supercritical case. Moreover, the nonexistence of minimizers in problem (1.7) for $d=0, p=q=1$ and small $\varepsilon$ holds for any doubly connected domain (with any capacity). (For $\operatorname{cap}(A)<\pi$ the proof is presented in [9]; this proof can be easily generalized to $\operatorname{cap}(A) \geq \pi$.) 
We conclude the introduction by outlining the scheme of the proof of Theorem 3 which employs a comparison argument. Fix an integer $d>0$. First, we establish the existence of minimizers in problem 1.7 for $p=q=d$ by using the so-called Price Lemma [8] (see Lemma 9 below), the uniform lower energy bound from Lemma 18 and the upper bound from Lemma 16 , which is obtained by considering $S^{1}$-valued testing maps. We show that these minimizers (which belong to $\mathcal{J}_{d d}^{(d)}$ ) are vortexless. Next, we argue by induction on the parameter $æ(p, q)=|d-p|+|d-q|$. This parameter is naturally associated with the number of vortices-for example, for the above minimizers in $\mathcal{J}_{d d}^{(d)}$, we have $æ(d, d)=0$. Given an integer $K \geq 0$, we assume the existence of minimizers in problem (1.7) for $p, q$ such that $æ(p, q) \leq K$ and $p, q \leq d$ (the induction hypothesis) and prove the existence of minimizers for $p, q$ such that $æ(p, q)=K+1$ and $p, q \leq d$. The first step in the induction procedure (when $K=0$ ) is shown in Section 5 The key technical point there is to construct a testing map $v \in \mathcal{J}_{d(d-1)}^{(d)}$ such that

$$
E_{\varepsilon}(v)<E_{\varepsilon}\left(u_{0}\right)+\pi,
$$

where $u_{0}$ is a minimizer of 1.2 in $\mathcal{J}_{d d}^{(d)}$. This map $v$ is constructed by using the minimizer $u_{0}$ and Möbius conformal maps on the unit disk with a prescribed single zero near the boundary. Then, given a minimizing sequence $\left\{u^{(k)}\right\} \subset \mathcal{J}_{d(d-1)}^{(d)}$ of problem 1.7 for $p=d, q=d-1$, we have, by 2.7) from Lemma 9 and (1.17),

$$
\begin{aligned}
E_{\varepsilon}(u)+\pi(|d-\operatorname{deg}(u, \partial \omega)|+\mid d-1- & \operatorname{deg}(u, \partial \Omega) \mid) \\
& \leq \lim _{k \rightarrow \infty} E_{\varepsilon}\left(u^{(k)}\right)<E_{\varepsilon}\left(u_{0}\right)+\pi,
\end{aligned}
$$

where $u$ is a weak $H^{1}$-limit of $\left\{u^{(k)}\right\}$ (possibly a subsequence). Then we estimate the left hand side of 1.18 by the lower energy bound from Lemma 18 and the right hand side of (1.18) by the upper energy bound from Lemma 16 . Thus

$$
\begin{aligned}
I_{0}(d, A)+\pi(2|d-\operatorname{deg}(u, \partial \omega)|+|d-1-\operatorname{deg}(u, \partial \Omega)| & +|d-\operatorname{deg}(u, \partial \Omega)|) \\
& <I_{0}(d, A)+\frac{3}{2} \pi .
\end{aligned}
$$

This implies that $\operatorname{deg}(u, \partial \omega)=d$, and either $\operatorname{deg}(u, \partial \Omega)=d-1$ or $\operatorname{deg}(u, \partial \Omega)=d$. In view of $(1.18)$, the only possible case is actually $\operatorname{deg}(u, \partial \Omega)=d-1$, since otherwise $u \in \mathcal{J}_{d d}^{(d)}$ and therefore $E_{\varepsilon}(u) \geq E_{\varepsilon}\left(u_{0}\right)$, which contradicts $\sqrt{1.18}$. Thus $u \in \mathcal{J}_{d(d-1)}^{(d)}$ and $u$ is a minimizer in $\mathcal{J}_{d(d-1)}^{(d)}$. The proof of the existence of minimizers for $p=d-1$, $q=d$ is quite similar. So we have shown that the existence of minimizers for $æ(p, q)=0$ implies the existence of minimizers for $æ(p, q)=1$. In the general case, when passing from $æ(p, q) \leq K$ to $æ(p, q) \leq K+1$ in problem (1.7), we use the same idea but it is technically much more involved. It requires the asymptotic analysis as $\varepsilon \rightarrow 0$ of minimizers $u_{p q}$ of $(1.7)$ with $æ(p, q)=K$, which is carried out in Section 6. Based on the results of this asymptotic analysis, we construct testing maps $v \in \mathcal{J}_{p^{\prime} q^{\prime}}^{(d)}\left(p^{\prime}=p\right.$, $q^{\prime}=q-1$ or $\left.p^{\prime}=p, q^{\prime}=q-1\right)$ such that $E_{\varepsilon}(v)<E_{\varepsilon}\left(u_{p q}\right)+\pi$. This allows us to employ the arguments similar to the reasoning in the first step of the induction procedure to show the attainability of the infimum in (1.7). 


\section{Preliminaries}

Throughout the paper we use the following notations:

- The vectors $a=\left(a_{1}, a_{2}\right)$ are identified with complex numbers $a=a_{1}+i a_{2}$.

- $a \cdot b$ stands for the scalar product $a \cdot b=a_{1} b_{1}+a_{2} b_{2}=\frac{1}{2}(a \bar{b}+\bar{a} b)$.

- $a \times b$ stands for the vector product $a \times b=a_{1} b_{2}-a_{2} b_{1}=\frac{i}{2}(a \bar{b}-\bar{a} b)$.

- The orientation of simple (without self-intersecting) curves in $\mathbb{R}^{2}$ (in particular $\partial \omega$ and $\partial \Omega$ ) is assumed to be counterclockwise. If $\mathcal{L}$ is such a curve, $\tau$ stands for the unit tangent vector pointing counterclockwise, and $v$ is the outer unit normal vector such that $(\nu, \tau)$ is direct.

- If $h$ is a scalar function, then $\nabla^{\perp} h=\left(-\partial_{x_{2}} h, \partial_{x_{1}} h\right)$.

- $B_{r}(y)$ is the open disk of radius $r$ centered at $y, B_{r}(y)=\left\{x \in \mathbb{R}^{2} ;|x-y|<r\right\}$.

\subsection{Properties of the solutions of 1.1 1.3}

As shown in [7] by a bootstrap argument, any solution $u \in H^{1}\left(A ; \mathbb{R}^{2}\right)$ of problem 1.1 . 1.3 is sufficiently regular (e.g., $u \in C^{2}(\bar{A})$ if $A$ has a $C^{2}$ boundary). By the maximum principle we also have

Lemma 6. The function $\rho(x)=|u(x)|$ satisfies $\rho \leq 1$ in A.

Locally, away from its zeros, $u$ can be written as $u=\rho e^{i \phi}$ with a real-valued phase $\phi$. We will also frequently make use of the current potential $h$ related to the solution $u$ of (1.1, 1.3 by

$$
\left\{\begin{array}{l}
\nabla^{\perp} h=\left(u \times \partial_{x_{1}} u, u \times \partial_{x_{2}} u\right) \quad \text { in } A, \\
h=1 \quad \text { on } \partial \Omega .
\end{array}\right.
$$

Unlike the phase $\phi$, the function $h$ is defined globally on $A\left(\nabla^{\perp} \phi\right.$ is also defined globally), and

$$
\nabla h=-\rho^{2} \nabla^{\perp} \phi \quad \text { when } \rho>0 .
$$

The existence of a unique solution of problem 2.1 and its elementary properties are established in the following

Lemma 7. There exists a unique solution $h$ of problem 2.1]. Moreover $h=$ const on $\partial \omega$, and

$$
\begin{gathered}
\Delta h=2 \partial_{x_{1}} u \times \partial_{x_{2}} u \quad \text { in } A, \\
\operatorname{div}\left(\frac{1}{\rho^{2}} \nabla h\right)=0 \quad \text { when } \rho>0 .
\end{gathered}
$$

Proof. The vector field $F=\left(u \times \partial_{x_{1}} u, u \times \partial_{x_{2}} u\right)$ is divergence free. Indeed, since $u$ is a smooth solution of [1.1], we have $\operatorname{div} F=u \times \Delta u=0$ in $A$. It follows that for any simply connected domain $W \subset A$ there is a unique (up to an additive constant) function $\Phi$ solving $\nabla^{\perp} \Phi=F$ in $W$ (this is the well known Poincaré lemma). Such a local solution $\Phi$ can be extended to a (possibly multi-valued) solution on $A$. Thanks to the fact that $u$ 
satisfies 1.3 we have $\partial \Phi / \partial \tau=-F \cdot v=0$ on $\partial A$, i.e. $\Phi$ takes constant values on every connected component of the boundary. Thus $\Phi$ is actually a single-valued function. Then $h(x)=\Phi(x)-\Phi(\partial \Omega)+1$ is the unique solution of $(2.1)$.

The verification of 2.3 is straightforward, and 2.4 follows directly from 2.2.

We will also use the following result, which is valid for any solution of the GL equation (1.1) (not necessarily satisfying [1.3).

Lemma 8 ([26]). Let $u$ be a solution of the GL equation (1.1] such that $|u| \leq 1$ and $E_{\varepsilon}(u) \leq \Lambda$, where $\Lambda$ is independent of $\varepsilon$. Then

$$
1-|u(x)|^{2} \leq \frac{\varepsilon^{2} C}{\operatorname{dist}^{2}(x, \partial A)}
$$

and

$$
\left|D^{k} u(x)\right| \leq \frac{C_{k}}{\operatorname{dist}^{k}(x, \partial A)},
$$

where $C, C_{k}$ are independent of $\varepsilon$.

\subsection{Minimization among maps from $\mathcal{J}$ with prescribed degrees}

Any minimizer of $(1.2)$ in the set $\mathcal{J}_{p q}$ with prescribed integer degrees $p$ and $q$ is clearly a solution of 11.1,1.3). However, the existence of minimizers is a nontrivial problem. In [10, 19, 7, 8, 9] the minimization problem for the Ginzburg-Landau functional (1.2) in $\mathcal{J}_{11}$ was considered. In the case when $A$ is a circular annulus, it was observed in [10] that minimizers, if they exist, break the symmetry when the ratio of the outer and inner radii of the annulus exceeds a certain threshold. By contrast, in the case when this ratio is sufficiently close to 1 , the existence of a unique minimizer and its symmetry are shown in [19]. The techniques in both [10] and [19] relied on the circular symmetry of the domain. A more general approach based on the Price Lemma was proposed in [7, 8].

Lemma 9 (Price Lemma [8]). Let $\left\{u^{(k)}\right\} \subset \mathcal{J}_{p q}$ be a sequence that converges to $u$ weakly in $H^{1}\left(A ; \mathbb{R}^{2}\right)$. Then

$$
\liminf _{k} \frac{1}{2} \int_{A}\left|\nabla u^{(k)}\right|^{2} d x \geq \frac{1}{2} \int_{A}|\nabla u|^{2} d x+\pi(|p-\operatorname{deg}(u, \partial \omega)|+|q-\operatorname{deg}(u, \partial \Omega)|),
$$

or equivalently (by Sobolev embeddings),

$$
\liminf _{k} E_{\varepsilon}\left(u^{(k)}\right) \geq E_{\varepsilon}(u)+\pi(|p-\operatorname{deg}(u, \partial \omega)|+|q-\operatorname{deg}(u, \partial \Omega)|) .
$$

Several proofs in this work are based on this lemma. With the help of Lemma 9 it was shown in [8] that the infimum of $[1.2]$ in $\mathcal{J}_{11}$ is always attained when $\operatorname{cap}(A) \geq \pi$. It was also conjectured in [8] that when $\operatorname{cap}(A)<\pi$ and $\varepsilon$ is sufficiently small the weak limit of any minimizing sequence is not in the class of admissible maps, i.e. the global minimizer does not exist. In [9] this nonexistence conjecture was proved by a contradiction argument based on explicit energy bounds. 
While the existence/nonexistence of minimizers in $\mathcal{J}_{p q}$ for $p=q=1$ is nontrivial and the answer depends on $\operatorname{cap}(A)$ and also on $\varepsilon$, the case $p=0, q=1$ (and $p=1$, $q=0)$ is simple. Arguing as in [7] we can show that $\inf \left\{E_{\varepsilon}(u) ; u \in \mathcal{J}_{01}\right\}$ is never attained. Indeed, using the pointwise equality $\left|\nabla u_{\varepsilon}\right|^{2}=2 \partial_{x_{1}} u_{\varepsilon} \times \partial_{x_{2}} u_{\varepsilon}+4\left|\partial_{\bar{z}} u_{\varepsilon}\right|^{2}$ and the Jacobian degree formula we have

$$
\frac{1}{2} \int_{A}|\nabla u|^{2} d x \geq\left|\int_{A} \partial_{x_{1}} u \times \partial_{x_{2}} u d x\right|=\pi|\operatorname{deg}(u, \partial \Omega)-\operatorname{deg}(u, \partial \omega)|=\pi
$$

whenever $u \in \mathcal{J}_{01}$. On the other hand, by constructing an explicit minimizing sequence in the spirit of [10] (see also [7]), we have $\inf \left\{E_{\varepsilon}(u) ; u \in \mathcal{J}_{01}\right\}=\pi$. Thus, if there exists a minimizer $u \in \mathcal{J}_{01}$, then $u \in H^{1}\left(A ; S^{1}\right)$ and $u$ solves the GL equation 1.1 . Then $u$ must be a constant map, which contradicts that $u \in \mathcal{J}_{01}$.

\section{Properties of the approximate bulk degree}

The degree of the restriction of maps from $H^{1}\left(A ; S^{1}\right)$ to any smooth closed curve, in particular $\partial \Omega$, is preserved by the weak $H^{1}$-convergence. This follows from [34], or can be shown directly using integration by parts as in 3.2 below (note that, for any $S^{1}$-valued map $u, \operatorname{deg}(u, \partial \Omega)=\operatorname{deg}(u, \partial \omega)=\operatorname{deg}(u, \mathcal{L})$, where $\mathcal{L}$ is an arbitrary smooth simple curve in $A$ enclosing $\omega$ ). Thus we have the decomposition

$$
H^{1}\left(A ; S^{1}\right)=\bigcup_{d \in \mathbb{Z}}\left\{u \in H^{1}\left(A ; S^{1}\right) ; \operatorname{deg}(u, \partial \Omega)=d\right\}
$$

into disjoint sets, each of them being closed in the weak $H^{1}$-topology of $H^{1}\left(A ; S^{1}\right)$.

Fix $\Lambda>0$. In this section we consider maps $u \in H^{1}\left(A ; \mathbb{R}^{2}\right)$ in the level set

$$
E_{\varepsilon}^{\Lambda}=\left\{u ; E_{\varepsilon}(u) \leq \Lambda\right\} .
$$

We show that the approximate bulk degree $\operatorname{abdeg}(u)$ classifies maps $u \in E_{\varepsilon}^{\Lambda}$ similarly to the above classification 3.1 of $S^{1}$-valued maps. We now establish the following basic properties of $\operatorname{abdeg}(u)$ :

(a) $\operatorname{abdeg}(u, A)=\operatorname{deg}(u, \partial \Omega)$ if $u \in H^{1}\left(A ; S^{1}\right)$,

(b) $|\operatorname{abdeg}(u)-\operatorname{abdeg}(v)| \leq \frac{2}{\pi}\|V\|_{C^{1}(A)} \Lambda^{1 / 2}\|u-v\|_{L^{2}(A)}$ if $u, v \in E_{\varepsilon}^{\Lambda}$, where $V$ is defined in 1.9 ).

The property (b) plays a key role in our considerations. It implies that, unlike $\operatorname{deg}(u, \partial \Omega)$, $\operatorname{abdeg}(u, A)$ is preserved with respect to weak $H^{1}$-convergence in the following sense:

(c) If $u_{n} \rightarrow u$ in $H^{1}\left(A ; \mathbb{R}^{2}\right)$, then $\operatorname{abdeg}\left(u_{n}, A\right) \rightarrow \operatorname{abdeg}(u, A)$.

The first property follows directly from the definition 1.10 of abdeg $(u)$. Indeed, integrating by parts in 1.10 , we get

$$
\operatorname{abdeg}(u)=\frac{1}{2 \pi} \int_{\partial \Omega} u \times \frac{\partial u}{\partial \tau} d s-\frac{1}{\pi} \int_{A} \partial_{x_{1}} u \times \partial_{x_{2}} u V d x=\operatorname{deg}(u, \partial \Omega)
$$

for any $u \in H^{1}\left(A ; S^{1}\right)\left(\partial_{x_{1}} u \times \partial_{x_{2}} u=0\right.$ a.e. in $A$ since $|u|=1$ a.e. $)$. The property (b) of $\operatorname{abdeg}(u)$ is proved in the following 
Lemma 10. For any $u, v \in H^{1}\left(A ; \mathbb{R}^{2}\right)$ we have

$$
|\operatorname{abdeg}(u)-\operatorname{abdeg}(v)| \leq \frac{1}{\pi}\|V\|_{C^{1}(A)}\left(\left(E_{\varepsilon}(u)\right)^{1 / 2}+\left(E_{\varepsilon}(v)\right)^{1 / 2}\right)\|u-v\|_{L^{2}(A)} .
$$

Proof. Integrating by parts, we get

$$
\begin{aligned}
2 \pi(\operatorname{abdeg}(u)-\operatorname{abdeg}(v))= & \int_{A}(u-v) \times\left(\partial_{x_{2}} u \partial_{x_{1}} V-\partial_{x_{1}} u \partial_{x_{2}} V\right) d x \\
& +\int_{A} v \times\left(\partial_{x_{2}}(u-v) \partial_{x_{1}} V-\partial_{x_{1}}(u-v) \partial_{x_{2}} V\right) d x \\
= & \int_{A}(u-v) \times\left(\partial_{x_{2}} u \partial_{x_{1}} V-\partial_{x_{1}} u \partial_{x_{2}} V\right) d x \\
& +\int_{A}(u-v) \times\left(\partial_{x_{2}} v \partial_{x_{1}} V-\partial_{x_{1}} v \partial_{x_{2}} V\right) d x .
\end{aligned}
$$

Then the statement of the lemma follows from the Cauchy-Schwarz inequality.

The main consequence of properties (a) and (b) of the function $\operatorname{abdeg}(u)$ is

Proposition 11. For sufficiently small $\varepsilon, \operatorname{abdeg}(u)$ is close to integers uniformly in $u \in$ $E_{\varepsilon}^{\Lambda}$. More precisely,

(d) $\sup \operatorname{dist}(\operatorname{abdeg}(u), \mathbb{Z}) \rightarrow 0$ as $\varepsilon \rightarrow 0$.

$u \in E_{\varepsilon}^{\Lambda}$

Remark 12. This proposition implies that, for small $\varepsilon, \mathcal{J}_{p q} \cap E_{\varepsilon}^{\Lambda}$ admits a decomposition into disjoint sets parametrized by $d$ :

$$
\mathcal{J}_{p q} \cap E_{\varepsilon}^{\Lambda}=\bigcup_{d \in \mathbb{Z}}\left(\mathcal{J}_{p q}^{(d)} \cap E_{\varepsilon}^{\Lambda}\right)
$$

Before proving this fact we note that Proposition 11 immediately implies Proposition 2 stated in the Introduction.

Proof of Proposition 11] According to 3.2 and Lemma 10 we have

$\sup _{u \in E_{\varepsilon}^{\Lambda}} \operatorname{dist}(\operatorname{abdeg}(u), \mathbb{Z}) \leq \sup _{u \in E_{\varepsilon}^{\Lambda}} \inf _{v \in E_{0}^{\Lambda}}|\operatorname{abdeg}(u)-\operatorname{abdeg}(v)| \leq \frac{2}{\pi}\|V\|_{C^{1}} \Lambda^{1 / 2} \delta_{\varepsilon}$,

where $\delta_{\varepsilon}$ is the (nonsymmetric) distance

$$
\delta_{\varepsilon}:=\sup _{u \in E_{\varepsilon}^{\Lambda}} \operatorname{dist}_{L^{2}(A)}\left(u, E_{0}^{\Lambda}\right)
$$

between $E_{\varepsilon}^{\Lambda}$ and

$$
E_{0}^{\Lambda}=\left\{u \in H^{1}\left(A ; S^{1}\right) ; E_{0}(u)=\frac{1}{2} \int_{A}|\nabla u|^{2} d x \leq \Lambda\right\} .
$$


We now show that $\delta_{\varepsilon} \rightarrow 0$ as $\varepsilon \rightarrow 0$. In view of 3.3 this yields the desired result. Assume towards a contradiction that $\delta_{\varepsilon_{k}} \geq c>0$ for a sequence $\varepsilon_{k} \rightarrow 0$. By virtue of the Sobolev embeddings, the supremum in $(3.4)$ is attained on $E_{\varepsilon}^{\Lambda}$, i.e. $\delta_{\varepsilon}=\operatorname{dist}_{L^{2}(A)}\left(u_{\varepsilon}, E_{0}^{\Lambda}\right)$, where $E_{\varepsilon}\left(u_{\varepsilon}\right) \leq \Lambda$. We can extract a subsequence of $\left\{u_{\varepsilon_{k}}\right\}$, still denoted by $\left\{u_{\varepsilon_{k}}\right\}$, that converges to a map $u$ weakly in $H^{1}\left(A ; \mathbb{R}^{2}\right)$. Thanks to Sobolev embeddings $u_{\varepsilon_{k}} \rightarrow u$ strongly in $L^{2}(A)$ and we have $u \in H^{1}\left(A ; S^{1}\right)$, since $\int_{A}\left(\left|u_{\varepsilon}\right|^{2}-1\right)^{2} d x \leq 4 \Lambda \varepsilon^{2}$. Moreover, $E_{0}(u) \leq \Lambda$ by the lower weak semicontinuity of the Dirichlet integral. Thus $u \in E_{0}^{\Lambda}$ and $\delta_{\varepsilon_{k}} \leq\left\|u-u_{\varepsilon_{k}}\right\|_{L^{2}(A)} \rightarrow 0$.

The following lemma illustrates the relation of $\operatorname{abdeg}(u)$, which is not necessarily an integer, with the standard notion of degree over a closed curve. It provides a simple criterion for the constraint $\operatorname{abdeg}(u) \in[d-1 / 2, d+1 / 2]$ in $(1.7]$ to be satisfied in a particular case when $u$ is a solution of equation 1.1 .

Lemma 13. Let $\mathcal{L}=\{x \in A ; V(x)=1 / 2\}$ denote the $1 / 2$ level set of $V$, where $V$ is the solution of 1.9 . ( $\mathcal{L}$ is a smooth curve enclosing $\omega$.) If a solution $u$ of the GL equation (1.1) satisfies $|u| \leq 1$ in $A$ and $E_{\varepsilon}(u) \leq \Lambda$, then

(i) $|u| \geq 1 / 2$ on $\mathcal{L}$,

(ii) we have

$$
\operatorname{abdeg}(u) \in[d-1 / 2, d+1 / 2] \Leftrightarrow \operatorname{deg}(u /|u|, \mathcal{L})=d
$$

when $\varepsilon \leq \varepsilon_{1}$, where $\varepsilon_{1}=\varepsilon_{1}(\Lambda)>0$ does not depend on $u$.

Remark 14. The choice of $\mathcal{L}$ in Lemma 13 is not unique. In fact, $\mathcal{L}$ can be any smooth curve that encloses $\omega$ and lies strictly inside the domain.

Proof. Consider the domain

$$
A_{\delta}=\{x \in A ; \delta<V(x)<1-\delta\},
$$

where $0<\delta<1 / 2$. Lemma 8 implies that $u$ satisfies

$$
|u| \geq 1 / 2 \quad \text { on } A_{\delta}
$$

when $\varepsilon<\varepsilon_{1}^{\prime}\left(\varepsilon_{1}^{\prime}=\varepsilon_{1}^{\prime}(\delta)>0\right)$. This proves (i). We can now write $u=\rho e^{i \psi}(\rho=|u|>$ $1 / 2$ ) on $A_{\delta}$, and find an extension of $\psi$ onto the entire domain $A$. To this end we will consider a conformal image of $A$.

It is well known (see, e.g., [1]) that there is a conformal mapping $\mathcal{G}$ of $A$ onto the annulus $\mathcal{O}$ with $R=\exp (\pi / \operatorname{cap}(A))$ and $1 / R$ as the outer and inner radii, respectively. Moreover, $\mathcal{G}$ is explicitly given by $\mathcal{G}=\exp \left(\frac{2 \pi}{\operatorname{cap}(A)}(V-1 / 2+i \Psi)\right)$, where $\Psi$ is a (multivalued) harmonic conjugate of $V$. Hence $\mathcal{G}$ maps $A_{\delta}$ onto the annulus $\mathcal{G}\left(A_{\delta}\right) \subset \mathcal{O}$ whose outer and inner radii are $R^{\prime}=\exp \left(\frac{2 \pi}{\operatorname{cap}(A)}(1 / 2-\delta)\right)$ and $1 / R^{\prime}$, respectively.

Now consider $\hat{\psi}(x)=\psi\left(\mathcal{G}^{-1}(x)\right)$ on $\mathcal{G}\left(A_{\delta}\right)$. We can extend $\hat{\psi}$ to the entire domain $\mathcal{O}$ by reflections $\hat{\psi}(x):=\hat{\psi}\left(x\left(R^{\prime}\right)^{2} /|x|^{2}\right)$ when $|x| \geq R^{\prime}$ and $\hat{\psi}(x):=\hat{\psi}\left(x /\left(R^{\prime}|x|\right)^{2}\right)$ when $|x| \leq 1 / R^{\prime}$. Thus

$$
\int_{\mathcal{O}}|\nabla \hat{\psi}|^{2} d x \leq \int_{\mathcal{G}\left(A_{\delta}\right)}|\nabla \hat{\psi}|^{2} d x+\int_{\mathcal{O} \backslash \mathcal{G}\left(A_{\delta}\right)}|\nabla \hat{\psi}|^{2} d x \leq 2 \int_{\mathcal{G}\left(A_{\delta}\right)}|\nabla \hat{\psi}|^{2} d x
$$


when $0<\delta<1 / 4$. Then (3.8), the conformal invariance of the Dirichlet integral and (3.7) imply

$$
\begin{aligned}
\int_{\mathcal{O}}|\nabla \hat{\psi}|^{2} d x & \leq 2 \int_{\mathcal{G}\left(A_{\delta}\right)}|\nabla \hat{\psi}|^{2} d x=2 \int_{A_{\delta}}|\nabla \psi|^{2} d x \\
& \leq 8 \int_{A_{\delta}} \rho^{2}|\nabla \psi|^{2} d x \leq 16 E_{\varepsilon}(u) \leq 16 \Lambda
\end{aligned}
$$

The desired extension of $\psi$ onto $A$ is now given by $\tilde{\psi}(x)=\hat{\psi}(\mathcal{G}(x))$. Using the conformal invariance of the Dirichlet integral again, we see by 3.9 that $E_{\varepsilon}\left(e^{i \tilde{\psi}}\right)=$ $\frac{1}{2} \int_{A}|\nabla \tilde{\psi}|^{2} d x=\frac{1}{2} \int_{\mathcal{O}}|\nabla \hat{\psi}|^{2} d x \leq 8 \Lambda$. Moreover, since $\tilde{\psi}=\psi$ on $A_{\delta}$ and $\rho=|u| \leq 1$, we obtain

$$
\begin{aligned}
\left\|u-e^{i \tilde{\psi}}\right\|_{L^{2}(A)}^{2} & =\int_{A \backslash A_{\delta}}\left|u-e^{i \tilde{\psi}}\right|^{2} d x+\int_{A_{\delta}}(\rho-1)^{2} d x \\
& \leq 4\left|A \backslash A_{\delta}\right|+\int_{A_{\delta}}\left(\rho^{2}-1\right)^{2} d x \leq 4\left(\left|A \backslash A_{\delta}\right|+\Lambda \varepsilon^{2}\right) .
\end{aligned}
$$

Then, by choosing small $\delta$ and $\varepsilon_{1}=\varepsilon_{1}(\delta)>0\left(\varepsilon_{1}(\delta)<\varepsilon_{1}^{\prime}\right)$, in view of Lemma 10 , the bounds $E_{\varepsilon}\left(e^{i \tilde{\psi}}\right) \leq 8 \Lambda, E_{\varepsilon}(u) \leq \Lambda$ and $\sqrt{3.10}$, we get $\left|\operatorname{abdeg}(u)-\operatorname{abdeg}\left(e^{i \tilde{\psi}}\right)\right|<1 / 2$, when $\varepsilon<\varepsilon_{1}$. But $\operatorname{abdeg}\left(e^{i \tilde{\psi}}\right)=\operatorname{deg}\left(e^{i \psi}, \partial \Omega\right)=\operatorname{deg}\left(e^{i \tilde{\psi}}, \mathcal{L}\right)=\operatorname{deg}(u /|u|, \mathcal{L})$, due to 3.2. Therefore if $\operatorname{deg}(u /|u|, \mathcal{L})=d$, then $\operatorname{abdeg}(u) \in[d-1 / 2, d+1 / 2]$, and vice versa. Thus (ii) is proved.

\section{Minimization among $S^{1}$-valued maps. Upper and lower bounds for problem (1.7)}

Consider the minimization problem

$$
I_{0}\left(d, A^{\prime}\right):=\inf \left\{E_{0}(u) ; u \in H^{1}\left(A^{\prime} ; S^{1}\right), \operatorname{deg}\left(u, \partial \omega^{\prime}\right)=\operatorname{deg}\left(u, \partial \Omega^{\prime}\right)=d\right\},
$$

where $E_{0}(u)=\int_{A^{\prime}}|\nabla u|^{2} d x, A^{\prime}=\Omega^{\prime} \backslash \overline{\omega^{\prime}}$, and $\omega^{\prime}, \Omega^{\prime}$ are arbitrary smooth bounded simply connected domains in $\mathbb{R}^{2}$ such that $\overline{\omega^{\prime}} \subset \Omega^{\prime}$. This problem is a particular case of the minimization problem considered in [11, Chapter I].

Proposition 15 ([11]). There exists a unique (up to multiplication by constants with unit modulus) solution $u$ of the minimization problem (4.1), and $u$ is a regular harmonic map in $A^{\prime}$ (i.e. $-\Delta u=u|\nabla u|^{2}$ in $\left.A^{\prime}, u \in H^{1}\left(A^{\prime}\right)\right)$ satisfying $u \times \frac{\partial u}{\partial v}=0$ on $\partial A^{\prime}$.

If $A^{\prime}=A$, then any minimizer $u$ of 4.1 belongs to $\mathcal{J}_{d d}$. By 3.2 we also have $\operatorname{abdeg}(u)$ $=d$. This yields the following (optimal) bound for (1.7), in the case $p=q=d$.

Lemma 16. For any $\varepsilon>0$ we have $m_{\varepsilon}(d, d, d) \leq I_{0}(d, A)$. 
It is shown in [11, Chapter I] that $I_{0}(d, A)$ can be expressed by

$$
I_{0}(d, A)=\frac{1}{2} \int_{A}\left|\nabla h_{0}\right|^{2} d x,
$$

where $h_{0}$ is the unique solution of the linear problem

$$
\left\{\begin{array}{l}
\Delta h_{0}=0 \quad \text { in } A, \\
h_{0}=1 \text { on } \partial \Omega, \quad h_{0}=\text { const } \text { on } \partial \omega, \\
\int_{\partial \Omega} \frac{\partial h_{0}}{\partial \nu} d \sigma=2 \pi d .
\end{array}\right.
$$

The solution $V$ of (1.9) and $h_{0}$ are related via $h_{0}=1+2 \pi d(V-1) / \operatorname{cap}(A)$, where $\operatorname{cap}(A)$ stands for the $H^{1}$-capacity of $A$ (see, e.g., [25]). Thus $I_{0}(d, A)=2(\pi d)^{2} / \mathrm{cap}(A)$, and this clearly holds for any doubly connected domain $A^{\prime}$ in place of $A$. Therefore we have

Lemma 17. $I_{0}\left(d, A^{\prime}\right)$ depends continuously on cap $\left(A^{\prime}\right)$.

Using this simple result, we obtain the following lower bound for the GL energy of solutions $u \in \mathcal{J}$ of the equation 1.1 .

Lemma 18. There exists $\varepsilon_{2}>0$ such that for any solution $u \in \mathcal{J}_{\text {lm }}$ of the GL equation 1.1) satisfying $E_{\varepsilon}(u) \leq \Lambda$ and $\operatorname{abdeg}(u) \in(d-1 / 2, d+1 / 2)$, we have

$$
E_{\varepsilon}(u) \geq I_{0}(d, A)-\frac{\pi}{2}+\pi(|d-l|+|d-m|)
$$

whenever $\varepsilon<\varepsilon_{2}$ ( $\varepsilon_{2}$ depends only on $\Lambda$ ).

Proof. The maximum principle implies that $|u| \leq 1$ on $A$. As in Lemma 13 we consider the domain $A_{\delta}$ defined by 3.6 where $\delta<1 / 2$ is a positive parameter to be chosen later. Since $|u| \leq 1$ on $A$ we can apply Lemma 8 to get the bound

$$
|u| \geq 1-\varepsilon \quad \text { in } A_{\delta}
$$

for $\varepsilon<\varepsilon_{2}^{\prime}\left(\varepsilon_{2}^{\prime}=\varepsilon_{2}^{\prime}(\delta, \Lambda)>0\right)$. We introduce the map

$$
\tilde{u}=\frac{1}{1-\varepsilon} \begin{cases}u & \text { if }|u|<1-\varepsilon \\ (1-\varepsilon) u /|u| & \text { otherwise. }\end{cases}
$$

By $\left[4.5\right.$ we have $|\tilde{u}|=1$ on $A_{\delta}$ and, according to $\operatorname{Lemma} 13, \operatorname{deg}(\tilde{u}, \mathcal{L})=d$ when $\varepsilon<\min \left\{\varepsilon_{1}, \varepsilon_{2}^{\prime}\right\}$. Consequently, the degrees of $\tilde{u}$ on both connected components of $\partial A_{\delta}$ are equal to $d$, so that $\frac{1}{2} \int_{A_{\delta}}|\nabla \tilde{u}|^{2} d x \geq I_{0}\left(d, A_{\delta}\right)$ (cf. $\sqrt[4.1]{ }$ ). Therefore, using the obvious pointwise inequality $|\nabla \tilde{u}|^{2} \geq 2\left|\partial_{x_{1}} \tilde{u} \times \partial_{x_{2}} \tilde{u}\right|$ and integration by parts, we get

$$
\begin{aligned}
\frac{1}{2} \int_{A}|\nabla \tilde{u}|^{2} d x & \geq \frac{1}{2} \int_{A_{\delta}}|\nabla \tilde{u}|^{2} d x+\sum_{k=1,2}\left|\int_{A_{\delta}^{(k)}} \partial_{x_{1}} \tilde{u} \times \partial_{x_{2}} \tilde{u} d x\right| \\
& \geq I_{0}\left(d, A_{\delta}\right)+\pi(|d-l|+|d-m|),
\end{aligned}
$$


where $A_{\delta}^{(k)}(k=1,2)$ are the outer and the inner connected components of $A \backslash A_{\delta}$. On the other hand, it follows from (4.6) that $|u| \leq|\tilde{u}| \leq 1$. Therefore,

$$
E_{\varepsilon}(\tilde{u}) \leq \frac{1}{(1-\varepsilon)^{2}} E_{\varepsilon}(u)
$$

Bounds (4.7) and 4.8 yield 4.4 when $\delta$ is such that $I_{0}\left(d, A_{\delta}\right) \geq I_{0}(d, A)-\pi / 4$ (cf. Lemma 17) and $\varepsilon$ is sufficiently small.

\section{From a vortexless minimizer to one with a single vortex}

The main Theorem 3 is proved by induction on the "number of vortices" in minimizers. More precisely, given an integer $d>0$, we show the existence of minimizers of (1.7) for $p=q=d$, then move on to the case $p=d-1, q=d$ and $p=d, q=d-1$, etc. The key point of the proof is the induction step, when the degree changes by one on $\partial \omega$ or $\partial \Omega$. This change in degree results in an additional vortex in a minimizer. For arbitrary $p$ and $q$ satisfying the conditions of Theorem 3, this step is quite technical. This is why we consider here a particular case of transition from $p=q=d$ (no vortices) to $p=d$, $q=d-1$ (one vortex). The transition from $p=q=d$ to $p=d-1$ and $q=d$ is quite similar. We first establish

Lemma 19. Fix an integer $d>0$. Then for sufficiently small $\varepsilon, \varepsilon \leq \varepsilon_{3}$ with $\varepsilon_{3}>0$, the infimum $m_{\varepsilon}(d, d, d)$ in (1.7) is always attained, and $m_{\varepsilon}(d, d, d) \leq I_{0}(d, A)$.

Proof. Let $u$ be a weak $H^{1}$-limit of a minimizing sequence $\left\{u^{(k)}\right\} \subset \mathcal{J}_{d d}^{(d)}$. Since any minimizer $v$ of problem (1.14 is an admissible testing map for problem (1.7), such a minimizing sequence exists and by using Lemma 9 we obtain

$$
E_{\varepsilon}(u)+\pi(|l-d|+|m-d|) \leq \liminf _{k \rightarrow \infty} E_{\varepsilon}\left(u^{(k)}\right) \leq \frac{1}{2} \int_{A}|\nabla v|^{2} d x=I_{0}(d, A),
$$

where $l=\operatorname{deg}(u, \partial \omega), m=\operatorname{deg}(u, \partial \Omega)$. Due to Proposition 2, we have $\operatorname{abdeg}(u) \in$ $(d-1 / 2, d+1 / 2)$ when $\varepsilon<\varepsilon_{0}$, therefore the first variation of $[1.2)$ at $u$ vanishes, i.e. $u$ is a solution of equation (1.1). Indeed, thanks to Lemma 10 , we know that for any $w \in H_{0}^{1}\left(A ; \mathbb{R}^{2}\right)$ with sufficiently small $H^{1}$-norm, $u^{(k)}+w$ is an admissible testing map when $k$ is large. Hence $E_{\varepsilon}(u+w)-E_{\varepsilon}(u)=\lim _{k \rightarrow \infty}\left(E_{\varepsilon}\left(u^{(k)}+w\right)-E_{\varepsilon}\left(u^{(k)}\right)\right) \geq 0$ (where $\lim _{k \rightarrow \infty}$ denotes any subsequential limit), and we are done. Now, since $u$ is a solution of (1.1), we can apply Lemma 18. Namely, we substitute 4.4 in 5.1) to get

$$
|d-l|+|d-m| \leq 1 / 4
$$

for $\varepsilon<\min \left\{\varepsilon_{0}, \varepsilon_{2}\right\}$, i.e. $l=m=d$ (since $l, m$ and $d$ are integers). Thus the infimum in (1.7) for $p=q=d$ is always attained for sufficiently small $\varepsilon$.

Next, we perform the transition from the minimization problem 1.7 for $p=q=d$ to that for $p=d, q=d-1$ and show that $m_{\varepsilon}(d, d-1, d)$ is always attained when $\varepsilon$ is 
sufficiently small. This is done by comparing $m_{\varepsilon}(d, d-1, d)$ with the energy $E_{\varepsilon}(u)$ of a minimizer $u$ of (1.7) for $p=q=d$. We first describe the properties of such minimizers.

In Section 6, it is shown that for small $\varepsilon$ any minimizer $u$ of $(1.7)$ for $p=q=d$ is vortexless (see Remark 24, i.e. $u=\rho e^{i \psi}$ with smooth $\rho>0$ and $\psi: A \rightarrow \mathbb{R} / 2 \pi d \mathbb{Z}$ (torus). It follows that we can write $u=\rho e^{i d \theta}$, where $e^{i \theta}$ and $\nabla \theta$ are smooth maps defined globally on $A$. Then the boundary value problem $1.1,1.3$ can be rewritten in terms of $\rho$ and $\theta$ as follows:

$$
\begin{gathered}
\left\{\begin{array}{l}
\operatorname{div}\left(\rho^{2} \nabla \theta\right)=0 \text { in } A, \\
\frac{\partial \theta}{\partial v}=0 \text { on } \partial A,
\end{array}\right. \\
\left\{\begin{array}{l}
-\Delta \rho+d^{2}|\nabla \theta|^{2} \rho+\frac{1}{\varepsilon^{2}} \rho\left(\rho^{2}-1\right)=0 \text { in } A, \\
\rho=1 \text { on } \partial A .
\end{array}\right.
\end{gathered}
$$

In view of 2.2, we also have

$$
\nabla h=-d \rho^{2} \nabla^{\perp} \theta \quad \text { in } A,
$$

where $h$ is the solution of (2.1). It follows that $(h, \theta)$ defines orthogonal local coordinates in a neighborhood of $\partial \Omega$, thus straightening out the boundary. Indeed, it is straightforward to verify that

$$
1-h(\partial \omega)=\frac{1}{\operatorname{cap}(A)} \int_{A} \nabla h \cdot \nabla V d x=2 \pi \operatorname{abdeg}(u) / \operatorname{cap}(A),
$$

while abdeg $(u) \geq d-1 / 2>0$. Then, by applying the maximum principle to 2.4 we get $1>h(x)>h(\partial \omega)$ in $A$. This in turn implies, by Hopf's lemma, that $\partial h / \partial v>0$ on $\partial \Omega$; i.e. the map $(h, \theta): A \rightarrow \mathbb{R} \times(\mathbb{R} / 2 \pi \mathbb{Z})$ can be extended to a $C^{1}$-diffeomorphism of a one-sided neighborhood of $\partial \Omega$ onto its image. Thus, there are some $\delta>0$ and a domain $G_{\delta} \subset A$ such that

$$
x \in G_{\delta} \mapsto(h, \theta) \in \Pi_{\delta}=(1-\delta, 1) \times(\mathbb{R} / 2 \pi \mathbb{Z})
$$

is a one-to-one correspondence which extends to a $C^{1}$-diffeomorphism of $\bar{G}_{\delta}$ onto $[1-\delta, 1] \times(\mathbb{R} / 2 \pi \mathbb{Z})$.

The following proposition is crucial for the existence of minimizers of (1.7) for $p=d, q=d-1$. In particular, if combined with Lemma 16, it provides a bound for $m_{\varepsilon}(d, d-1, d)$ independent of $\varepsilon$.

Proposition 20. Let $u=\rho e^{i d \theta}, \rho>0$, be a minimizer of 1.7 for $p=q=d$. Assume that $\varepsilon$ is small enough that Proposition 2 holds with $\Lambda=I_{0}(d, A)$. Then there is a testing map $v \in \mathcal{J}_{d(d-1)}$ such that $\operatorname{abdeg}(v) \in(d-1 / 2, d+1 / 2)$ and

$$
E_{\varepsilon}(v)-E_{\varepsilon}(u)<\pi
$$


In Section 7 a generalized version of Proposition 20 is used to show the existence of minimizers with several vortices.

Proof of Proposition 20 We seek a testing map $v$ of the form

$$
v=\rho w_{t},
$$

where $w_{t} \in \mathcal{J}_{d(d-1)}$ will be defined below. The following lemma allows us to compare the energy $E_{\varepsilon}(u)$ of $u$ with that of $v$.

Lemma 21. If $w \in H^{1}\left(G^{\prime} ; \mathbb{R}^{2}\right), G^{\prime} \subset A$, is such that $|w|=1$ on $\partial G^{\prime}$, then

$$
\begin{aligned}
\int_{G^{\prime}}\left(|\nabla(\rho w)|^{2}+\frac{1}{2 \varepsilon^{2}}\left(|\rho w|^{2}-1\right)^{2}\right) d x & \\
& =\int_{G^{\prime}}\left(|\nabla u|^{2}+\frac{1}{2 \varepsilon^{2}}\left(|u|^{2}-1\right)^{2}\right) d x+2 L_{\varepsilon}^{(d)}\left(w, G^{\prime}\right)
\end{aligned}
$$

where

$$
\begin{aligned}
L_{\varepsilon}^{(d)}\left(w, G^{\prime}\right)= & \frac{1}{2} \int_{G^{\prime}} \rho^{2}|\nabla w|^{2} d x-\frac{d^{2}}{2} \int_{G^{\prime}}|\nabla \theta|^{2} \rho^{2}|w|^{2} d x \\
& +\frac{1}{4 \varepsilon^{2}} \int_{G^{\prime}} \rho^{4}\left(|w|^{2}-1\right)^{2} d x .
\end{aligned}
$$

This result is a version of the factorization argument from [14]; its proof is presented at the end of this section.

Note that if $G^{\prime}=G_{\delta}$ we can rewrite the functional 5.8 by using local coordinates $(h, \theta)$ as (cf. 5.5)

$$
\begin{aligned}
L_{\varepsilon}^{(d)}\left(w, G_{\delta}\right)= & \frac{d}{2} \int_{\Pi_{\delta}}\left|\partial_{h} w\right|^{2} \rho^{4} d h d \theta+\frac{1}{2 d} \int_{\Pi_{\delta}}\left(\left|\partial_{\theta} w\right|^{2}-d^{2}|w|^{2}\right) d h d \theta \\
& +\frac{1}{4 \varepsilon^{2}} \int_{\Pi_{\delta}} \rho^{2}\left(|w|^{2}-1\right)^{2} \frac{d h d \theta}{d|\nabla \theta|^{2}}
\end{aligned}
$$

Instead of dealing with $L_{\varepsilon}^{(d)}\left(w, G_{\delta}\right)$ we will make use of simplified functional with a quadratic penalty term and parameter $\lambda=\lambda(\varepsilon, d)$ to be determined in 5.14 below,

$$
\begin{aligned}
M_{\lambda}(w)= & \frac{1}{2 d} \int_{\Pi_{\delta}}\left(d^{2}\left|\partial_{h} w\right|^{2}+\left|\partial_{\theta} w\right|^{2}\right) d h d \theta \\
& +\frac{1}{2 d} \int_{\Pi_{\delta}}\left(\lambda\left|w-e^{i d \theta}\right|^{2}-d^{2}|w|^{2}\right) d h d \theta
\end{aligned}
$$

Note also that the integrand in the first term of $L_{\varepsilon}^{(d)}\left(w, G_{\delta}\right)$ has been multiplied here by the factor $\rho^{-4} \geq 1$. The functional 5.10 can be simplified using separation of variables. 
Now consider the map $w_{t}$ that is given by $w_{t}=e^{i d \theta}$ in $A \backslash G_{\delta}$, and extended to $G_{\delta}$ as a minimizer of the functional $M_{\lambda}(w)$, with the following prescribed boundary data:

$$
\begin{aligned}
& w_{t}=e^{i d \theta} \mathcal{F}_{t}\left(e^{i \theta}\right) \quad \text { on } \partial \Omega, \\
& w_{t}=e^{i d \theta} \quad \text { on } \partial G_{\delta} \backslash \partial \Omega,
\end{aligned}
$$

Here $\lambda \geq 2 d^{2}, \mathcal{F}_{t}(z):=\mathcal{C}_{t}(\bar{z})$ (where the bar stands for the complex conjugate), $\mathcal{C}_{t}(z)=$ $\frac{z-(1-t)}{z(1-t)-1}$ is the classical Möbius conformal map from the unit disk onto itself, and $t<1$ is a positive parameter to be determined later. Since $\operatorname{deg}\left(\mathcal{F}_{t}, S^{1}\right)=-1$ and $\operatorname{deg}\left(e^{i \theta}, \partial \Omega\right)$ $=1$, the standard properties of the topological degree imply that if $v$ is defined by (5.7), then

$$
\operatorname{deg}(v, \partial \Omega)=d-1, \quad \operatorname{deg}(v, \partial \omega)=d .
$$

The map $w_{t}$ is well defined, because the functional $M_{\lambda}(w)$ with Dirichlet condition on the boundary has a unique minimizer for $\lambda \geq 2 d^{2}$. Moreover $\left|w_{t}\right| \leq 2$. Otherwise by taking $\tilde{w}_{t}=w_{t} \min \left\{1,2 /\left|w_{t}\right|\right\}$ in place of $w_{t}$, the first term in 5.10 does not increase while the second decreases, i.e. $M_{\lambda}\left(\tilde{w}_{t}\right)<M_{\lambda}\left(w_{t}\right)$; a contradiction.

Note that under the choice

$$
\lambda:=\max \left\{\frac{9}{2 \varepsilon^{2} \inf _{G_{\delta}}|\nabla \theta|^{2}}, 2 d^{2}\right\}
$$

$\left(|\nabla \theta|>0\right.$ on the closure of $\left.G_{\delta}\right)$ we have

$$
\begin{aligned}
\rho^{2}\left(\left|w_{t}\right|^{2}-1\right)^{2} & \leq\left(\left|w_{t}\right|-1\right)^{2}\left(\left|w_{t}\right|+1\right)^{2} \leq\left|w_{t}-e^{i d \theta}\right|^{2}\left(\left|w_{t}\right|+1\right)^{2} \\
& \leq 9\left|w_{t}-e^{i d \theta}\right|^{2} \leq 2 \varepsilon^{2} \lambda|\nabla \theta|^{2}\left|w_{t}-e^{i d \theta}\right|^{2} \quad \text { in } G_{\delta}
\end{aligned}
$$

thanks to the bounds $\left|w_{t}\right| \leq 2$ and $\rho \leq 1$. It follows that $L_{\varepsilon}^{(d)}\left(w_{t}, G_{\delta}\right) \leq M_{\lambda}\left(w_{t}\right)$. We now see, by virtue of Lemma 21 , that $v=\rho w_{t}$ satisfies

$$
E_{\varepsilon}(v) \leq E_{\varepsilon}(u)+M_{\lambda}\left(w_{t}\right) .
$$

We next obtain a representation for $M_{\lambda}\left(w_{t}\right)$ through separation of variables. Namely, expanding $z^{d} \mathcal{F}_{t}(z)$ on $S^{1}$ as

$$
z^{d} \mathcal{F}_{t}(z)=(1-t) z^{d}+t(t-2) \sum_{k=0}^{\infty}(1-t)^{k} z^{d-k-1}
$$

we seek $w_{t}$ as a Fourier series

$$
w_{t}=\left(1-t f_{-1}(h)\right) e^{i d \theta}+t(t-2) \sum_{k=0}^{\infty}(1-t)^{k} f_{k}(h) e^{-i(k-d+1) \theta},
$$

where the coefficients $f_{k}(h)$ satisfy, according to 5.11, 5.12,

$$
f_{k}(1-\delta)=0, \quad f_{k}(1)=1 .
$$


We substitute 5.16 into 5.10 to obtain

$$
M_{\lambda}\left(w_{t}\right)=\frac{t^{2} \pi}{d} \Phi_{-1}\left(f_{-1}\right)+\frac{t^{2} \pi}{d} \sum_{k=0}^{\infty}(t-2)^{2}(1-t)^{2 k} \Phi_{k}\left(f_{k}\right),
$$

where

$$
\Phi_{k}\left(f_{k}\right)=\int_{1-\delta}^{1}\left(d^{2}\left|f_{k}^{\prime}(h)\right|^{2}+\left((k-d+1)^{2}+\lambda-d^{2}\right)\left|f_{k}(h)\right|^{2}\right) d h .
$$

Minimizing (5.19) under the conditions (5.17) we get

$$
f_{k}(h)=\frac{e^{k_{+}(h-1)}}{1-e^{\left(k_{-}-k_{+}\right) \delta}}+\frac{e^{k_{-}(h-1)}}{1-e^{\left(k_{+}-k_{-}\right) \delta}},
$$

where $k_{ \pm}= \pm \frac{1}{d} \sqrt{(k-d+1)^{2}+\lambda-d^{2}}$. Therefore,

$$
\Phi_{k}\left(f_{k}\right)=d(k-d+1)\left(1+\frac{\lambda-d^{2}}{2 k^{2}}+O\left(1 / k^{3}\right)\right) \quad \text { as } k \rightarrow \infty .
$$

Finally, using (5.21) in 5.18), we obtain

$$
\begin{aligned}
M_{\lambda}\left(w_{t}\right) & \leq \pi\left((1-t)^{2}-1\right)^{2} \sum_{k=0}^{\infty} k(1-t)^{2 k}+2 \pi t^{2}\left(\lambda-d^{2}\right) \sum_{k=1}^{\infty} \frac{(1-t)^{2 k}}{k}+C t^{2} \\
& =\pi\left(1-2 t-2 t^{2}\left(\lambda-d^{2}\right) \log \left(1-(1-t)^{2}\right)\right)+(C+\pi) t^{2}
\end{aligned}
$$

Observe that the right hand side of 5.22) is strictly less than $\pi$ when $t>0$ is chosen sufficiently small. By (5.15), for such $t$ the map $v=\rho w_{t}$ satisfies (5.6).

It remains only to show that $\operatorname{abdeg}(v) \in(d-1 / 2, d+1 / 2)$. To this end note that by 5.16 and 5.20,,$w_{t} \rightarrow e^{i d \theta}$ pointwise in $G_{\delta}$ as $t \rightarrow 0$. Therefore $\rho w_{t} \rightarrow \rho e^{i d \theta}(=u)$ weakly in $H^{1}(A)$, so that $\operatorname{abdeg}\left(\rho w_{t}\right) \rightarrow \operatorname{abdeg}(u)$. On the other hand, by Proposition 2 . we know that $d-1 / 2<\operatorname{abdeg}(u)<d+1 / 2$. Thus, after possibly passing to a smaller $t$, $v=\rho w_{t}$ satisfies the required property.

Now, under the conditions of Proposition 20, there exists a minimizing sequence $\left\{u^{(k)}\right\} \subset \mathcal{J}_{d(d-1)}^{(d)}$ of testing maps from problem 1.7 for $p=d, q=d-1$ such that $\lim _{k \rightarrow \infty} E_{\varepsilon}\left(u^{(k)}\right)<m_{\varepsilon}(d, d, d)+\pi$ and $u^{(k)}$ weakly $H^{1}$-converges to a map $u \in \mathcal{J}$. Moreover, any minimizing sequence has a subsequence with the same properties. We show that any weak limit $u$ is also an admissible map. Let $l=\operatorname{deg}(u, \partial \omega)$ and $m=\operatorname{deg}(u, \partial \Omega)$. By virtue of Lemma 18 (in the same way as in Lemma 19 one shows that $u$ satisfies (1.1) ) and Lemma 9 we have

$$
\begin{aligned}
& I_{0}(d, A)-\frac{\pi}{2}+ \pi(2|d-l|+|d-1-m|+|d-m|) \\
& \leq E_{\varepsilon}(u)+\pi(|d-l|+|d-1-m|)<m_{\varepsilon}(d, d, d)+\pi,
\end{aligned}
$$


since $\operatorname{abdeg}(u)=\lim _{k \rightarrow \infty} \operatorname{abdeg}\left(u^{(k)}\right) \in[d-1 / 2, d+1 / 2]$. Due to Lemma 19, we have $m_{\varepsilon}(d, d, d) \leq I_{0}(d, A)$ so that 5.23 implies that $l=d$ and either $m=d-1$ or $m=d$. In the latter case, $u$ becomes an admissible map in problem (1.7) for $p=q=d$ and therefore $E_{\varepsilon}(u) \geq m_{\varepsilon}(d, d, d)$, which contradicts the last inequality in (5.23). Thus, $u \in \mathcal{J}_{d(d-1)}, \operatorname{abdeg}(u) \in[d-1 / 2, d+1 / 2]$, i.e. $u$ is in the set $\mathcal{J}_{d(d-1)}^{(d)}$ of admissible testing maps of problem (1.7) for $p=d, q=d-1$.

Proof of Lemma 21] We have, using (5.4),

$$
\begin{aligned}
\int_{G^{\prime}}|\nabla(\rho w)|^{2} d x= & \int_{G^{\prime}}\left(\rho^{2}|\nabla w|^{2}+\nabla \rho \cdot \nabla\left(\rho\left(|w|^{2}-1\right)\right)+|\nabla \rho|^{2}\right) d x \\
= & \int_{G^{\prime}}\left(\rho^{2}|\nabla w|^{2}+d^{2} \rho^{2}|\nabla \theta|^{2}+\frac{1}{\varepsilon^{2}} \rho^{2}\left(\rho^{2}-1\right)\right) d x \\
& -\int_{G^{\prime}}\left(d^{2} \rho^{2}|\nabla \theta|^{2}|w|^{2}+\frac{1}{\varepsilon^{2}} \rho^{2}\left(\rho^{2}-1\right)|w|^{2}-|\nabla \rho|^{2}\right) d x .
\end{aligned}
$$

Then simple algebraic manipulations yield the required result.

\section{Asymptotic behavior of local minimizers}

In the previous section, we established the existence of minimizers of 1.2 in $\mathcal{J}_{d d}^{(d)}$ and demonstrated the first induction step of the proof of Theorem 3 that involves a transition from $p=q=d$ to $p=d, q=d-1$ in (1.7). (In fact, modulo the assumption that minimizers in $\mathcal{J}_{d d}^{(d)}$ are vortexless, we actually proved the existence of minimizers in $\mathcal{J}_{(d-1) d}^{(d)}$ and $\mathcal{J}_{d(d-1)}^{(d)}$.) In order to show the induction step for any integer $p \leq d$ and $q \leq d$, we need to establish some properties of minimizers of 1.7 . We are especially interested in their behavior near the boundary. At this point, we assume we are given a family $\left\{u_{\varepsilon}\right\}$ of minimizers for 1.7 and

$$
E_{\varepsilon}\left(u_{\varepsilon}\right) \leq \Lambda:=I_{0}(d, A)+\pi(|d-p|+|d-q|) .
$$

We suppose also that $\varepsilon \leq \varepsilon_{0}$, where $\varepsilon_{0}=\varepsilon_{0}(\Lambda)>0$ as in Proposition 2 It follows that the maps $u_{\varepsilon}$ are local minimizers of $E_{\varepsilon}(u)$ in $\mathcal{J}$ and therefore satisfy $1.1,1.3$.

Throughout this section we will use the notation $\rho_{\varepsilon}(x)=\left|u_{\varepsilon}(x)\right|$, the function $h_{\varepsilon}(x)$ that is the unique solution of 2.1) (associated with $u_{\varepsilon}$ ), and the contour $\mathcal{L}$ as in Lemma 13 The contour $\mathcal{L}$ separates the two open subdomains $Q^{ \pm}$in $A$, where $Q^{+}$is the domain enclosed by $\partial \Omega$ and $\mathcal{L}$, and $Q^{-}=A \backslash\left(Q^{+} \cup \mathcal{L}\right)$. We also set $Q_{\varepsilon}^{ \pm}=\left\{x \in Q^{ \pm} ; \rho_{\varepsilon}^{2}(x) \leq\right.$ $\left.1-\varepsilon^{1 / 2}\right\}$.

\subsection{Proof of Theorem 4}

Since $\left|\nabla h_{\varepsilon}\right| \leq\left|\nabla u_{\varepsilon}\right|$ (by Lemma 6 , the family $\left\{h_{\varepsilon}\right\}$ is bounded in $H^{1}(A)$, and therefore there is a sequence $\varepsilon_{k} \rightarrow 0$ such that

$$
h_{\varepsilon_{k}} \rightarrow h \quad \text { weakly in } H^{1}(A) \text { as } k \rightarrow \infty .
$$


In order to identify $h$, we make use of Lemma 8 to deduce that, up to a subsequence, the maps $u_{\varepsilon_{k}}$ converge to an $S^{1}$-valued map $u$ in $C_{\text {loc }}^{1}(A)$. Since $\partial_{x_{1}} u \times \partial_{x_{2}} u=0$ a.e. in $A$, we have $\Delta h_{\varepsilon_{k}}=2 \partial_{x_{1}} u_{\varepsilon_{k}} \times \partial_{x_{2}} u_{\varepsilon_{k}} \rightarrow 0$ in $C_{\text {loc }}^{0}(A)$, thus $h$ is a harmonic function. Moreover, $h=1$ on $\partial \Omega$ and $h=$ const on $\partial \omega$. On the other hand,

$$
\operatorname{abdeg}\left(u_{\varepsilon_{k}}\right)=\frac{1}{2 \pi} \int_{A} \nabla h_{\varepsilon_{k}} \cdot \nabla V d x \rightarrow \frac{1}{2 \pi} \int_{A} \nabla h \cdot \nabla V d x=\frac{1}{2 \pi} \int_{\partial \Omega} \frac{\partial h}{\partial \nu} d s .
$$

According to property (d) of abdeg ( $)$ ) (see Proposition 11 in Section 3), $\operatorname{abdeg}\left(u_{\varepsilon}\right) \rightarrow d$ as $\varepsilon \rightarrow 0$. Therefore $h=h_{0}$ (where $h_{0}$ is the unique solution of (4.3) ) and the convergence in 6.2 holds for the whole family $\left\{h_{\varepsilon}\right\}$. Thus, applying Lemma 8 again, we obtain

$$
h_{\varepsilon} \rightarrow h_{0} \quad \text { in } C_{\text {loc }}^{1}(A) \text { and weakly in } H^{1}(A) \text { as } \varepsilon \rightarrow 0 .
$$

By (6.1) and Lemma 8 , the maps $u_{\varepsilon}$ converge, up to a subsequence, to $u \in H^{1}\left(A ; S^{1}\right)$ in $C_{\text {loc }}^{1}(A)$ and weakly in $H^{1}(A)$. Moreover, $\operatorname{abdeg}(u)=d$ and in view of $\langle 6.3|,| \nabla u|=$ $\left|\nabla h_{0}\right|$ a.e. in $A$. It follows that $u$ is the solution of the minimization problem (1.14).

In order to demonstrate the energy expansion stated in Theorem 4 , we argue as follows: by using the pointwise equalities $\left|\nabla u_{\varepsilon}\right|^{2}=2 \partial_{x_{1}} u_{\varepsilon} \times \partial_{x_{2}} u_{\varepsilon}+4\left|\partial_{\bar{z}} u_{\varepsilon}\right|^{2}$ and $\left|\nabla u_{\varepsilon}\right|^{2}=$ $-2 \partial_{x_{1}} u_{\varepsilon} \times \partial_{x_{2}} u_{\varepsilon}+4\left|\partial_{z} u_{\varepsilon}\right|^{2}$ and the pointwise inequality $\left|\nabla u_{\varepsilon}\right| \geq\left|\nabla h_{\varepsilon}\right|$, we have

$$
\begin{aligned}
\frac{1}{2} \int_{A}\left|\nabla u_{\varepsilon}\right|^{2} d x \geq & -\int_{Q_{\varepsilon}^{+}} \partial_{x_{1}} u_{\varepsilon} \times \partial_{x_{2}} u_{\varepsilon} d x+2 \int_{Q_{\varepsilon}^{+}}\left|\partial_{z} u_{\varepsilon}\right|^{2} d x+\int_{Q_{\varepsilon}^{-}} \partial_{x_{1}} u_{\varepsilon} \times \partial_{x_{2}} u_{\varepsilon} d x \\
& +2 \int_{Q_{\varepsilon}^{-}}\left|\partial_{\bar{z}} u_{\varepsilon}\right|^{2} d x+\frac{1}{2} \int_{A \backslash\left(Q_{\varepsilon}^{+} \cup Q_{\varepsilon}^{-}\right)}\left|\nabla h_{\varepsilon}\right|^{2} d x
\end{aligned}
$$

Let us estimate the right hand side of 6.4 from below. Introducing $\sigma_{\varepsilon}(x)=\max \left\{\rho_{\varepsilon}^{2}(x)\right.$, $\left.1-\varepsilon^{1 / 2}\right\}$, we have (by 2.3 , 2.4 )

$$
\operatorname{div}\left(\frac{1}{\sigma_{\varepsilon}(x)} \nabla h_{\varepsilon}\right)=\frac{2}{1-\varepsilon^{1 / 2}} \begin{cases}0 & \text { in } A \backslash\left(Q_{\varepsilon}^{+} \cup Q_{\varepsilon}^{-}\right), \\ \partial_{x_{1}} u_{\varepsilon} \times \partial_{x_{2}} u_{\varepsilon} & \text { otherwise. }\end{cases}
$$

Integrating 6.5 over $Q^{+}$, we get, for sufficiently small $\varepsilon$,

$$
\begin{aligned}
\frac{2}{1-\varepsilon^{1 / 2}} \int_{Q_{\varepsilon}^{+}} \partial_{x_{1}} u_{\varepsilon} & \times \partial_{x_{2}} u_{\varepsilon} d x=\int_{\partial \Omega} \frac{\partial h_{\varepsilon}}{\partial v} d s-\int_{\mathcal{L}} \frac{\partial h_{\varepsilon}}{\partial v} \frac{d s}{\rho_{\varepsilon}^{2}(x)} \\
= & \int_{\partial \Omega} u_{\varepsilon} \times \frac{\partial u_{\varepsilon}}{\partial \tau} d s-\int_{\mathcal{L}} \frac{u_{\varepsilon}}{\left|u_{\varepsilon}\right|} \times \frac{\partial}{\partial \tau} \frac{u_{\varepsilon}}{\left|u_{\varepsilon}\right|} d s=2 \pi(q-d),
\end{aligned}
$$

where we have used Lemmas 8 and 13 , Thus, we have

$$
\int_{Q_{\varepsilon}^{+}} \partial_{x_{1}} u_{\varepsilon} \times \partial_{x_{2}} u_{\varepsilon} d x=\left(1-\varepsilon^{1 / 2}\right) \pi(q-d) .
$$

Similarly, integrating 6.5 over $Q^{-}$we obtain

$$
\int_{Q_{\varepsilon}^{-}} \partial_{x_{1}} u_{\varepsilon} \times \partial_{x_{2}} u_{\varepsilon} d x=\left(1-\varepsilon^{1 / 2}\right) \pi(d-p) .
$$


In order to estimate the last term on the right hand side of (6.4), we write it as

$$
\begin{aligned}
\int_{A \backslash\left(Q_{\varepsilon}^{+} \cup Q_{\varepsilon}^{-}\right)}\left|\nabla h_{\varepsilon}\right|^{2} d x= & \int_{A \backslash\left(Q_{\varepsilon}^{+} \cup Q_{\varepsilon}^{-}\right)}\left|\nabla h_{\varepsilon}-\nabla h_{0}\right|^{2} d x+\int_{A}\left(2 \nabla h_{\varepsilon}-\nabla h_{0}\right) \cdot \nabla h_{0} d x \\
& -\int_{Q_{\varepsilon}^{+} \cup Q_{\varepsilon}^{-}}\left(2 \nabla h_{\varepsilon}-\nabla h_{0}\right) \cdot \nabla h_{0} d x,
\end{aligned}
$$

and note that by virtue of 6.1 , the measure of $Q_{\varepsilon}^{+} \cup Q_{\varepsilon}^{-}$vanishes as $\varepsilon \rightarrow 0$, so that

$$
\int_{A \backslash\left(Q_{\varepsilon}^{+} \cup Q_{\varepsilon}^{-}\right)}\left|\nabla h_{\varepsilon}\right|^{2} d x=\int_{A \backslash\left(Q_{\varepsilon}^{+} \cup Q_{\varepsilon}^{-}\right)}\left|\nabla h_{\varepsilon}-\nabla h_{0}\right|^{2} d x+\int_{A}\left|\nabla h_{0}\right|^{2} d x+o(1) .
$$

Thus 6.6 6.8, 6.4 6.1 imply $E_{\varepsilon}\left(u_{\varepsilon}\right) \rightarrow E_{0}(u)+\pi(|d-p|+|d-q|)$.

As a byproduct of the above proof, by 6.6) $6.8,6.4$ and 6.1 , we get

$$
\begin{gathered}
\int_{A}\left(\left|u_{\varepsilon}\right|^{2}-1\right)^{2} d x=o\left(\varepsilon^{2}\right), \\
\int_{A \backslash\left(Q_{\varepsilon}^{+} \cup Q_{\varepsilon}^{-}\right)}\left|\nabla h_{\varepsilon}-\nabla h_{0}\right|^{2} d x=o(1), \\
\int_{Q_{\varepsilon}^{+}}\left|\partial_{z} u_{\varepsilon}\right|^{2} d x=o(1), \quad \int_{Q_{\varepsilon}^{-}}\left|\partial_{\bar{z}} u_{\varepsilon}\right|^{2} d x=o(1) .
\end{gathered}
$$

6.2. Properties of minimizers of (1.7) for small $\varepsilon$

First, by using (6.9) and the methods from [11] we deduce that $\rho_{\varepsilon}$ converges to 1 uniformly on compacts in $A$. Moreover, we have

Lemma 22. For any $\mu>0$ we have

$$
\sup \left\{\operatorname{dist}(y, \partial A) ; y \in A, \rho_{\varepsilon}^{2}(y)<1-\mu\right\}=o(\varepsilon) .
$$

Proof. Assume towards a contradiction that for a sequence $\varepsilon_{k} \rightarrow 0$ and $\gamma>0$ we have $\rho_{\varepsilon_{k}}^{2}\left(y_{k}\right)<1-\mu$ and $\operatorname{dist}\left(y_{k}, \partial A\right) \geq \gamma \varepsilon_{k}$. Due to $2.6,\left.|\nabla| u_{\varepsilon_{k}}\right|^{2} \mid \leq \alpha / \varepsilon_{k}$ in $B_{\lambda \varepsilon_{k}}\left(y_{k}\right)$, where $0<\lambda<\gamma$ and $\alpha=\alpha(\lambda)$ is independent of $\varepsilon_{k}$. It follows that $\left|u_{\varepsilon_{k}}(x)\right|^{2}<$ $1-\mu+\delta \alpha$ when $x \in B_{\delta \varepsilon_{k}}\left(y_{k}\right)$ and $\delta<\lambda$. Then $B_{\delta \varepsilon_{k}}\left(y_{k}\right) \subset A$ and

$$
\frac{1}{\varepsilon_{k}^{2}} \int_{B_{\delta \varepsilon_{k}}\left(y_{k}\right)}\left(\left|u_{\varepsilon_{k}}\right|^{2}-1\right)^{2} d x \geq \pi(\mu-\delta \alpha)^{2} \delta^{2}>0,
$$

as soon as $0<\delta<\min \{\lambda, \mu /(2 \alpha)\}$. This contradicts 6.9.

Important properties of $u_{\varepsilon}$ and $h_{\varepsilon}$, in the vicinity of the boundary $\partial A$, are established in

Lemma 23. For any $0<\mu<1$ and $\kappa<1$ there are $\hat{\varepsilon}_{1}(\mu), \hat{\varepsilon}_{2}(\mu, \kappa)>0$ such that if $\rho_{\varepsilon}^{2}(y) \leq 1-\mu$, then

(a) for $\varepsilon<\hat{\varepsilon}_{1}(\mu)$ we have: $h_{\varepsilon}(y) \geq 1+\mu / 4$ if $\operatorname{dist}(y, \partial \Omega)<\varepsilon$ and $h_{\varepsilon}(y) \leq h_{\varepsilon}(\partial \omega)-\mu / 4$ if $\operatorname{dist}(y, \partial \omega)<\varepsilon$; 
(b) for $\varepsilon<\hat{\varepsilon}_{2}(\mu, \kappa)$ we have

$$
\frac{1}{2} \int_{A \cap B_{\varepsilon}(y)}\left|\nabla u_{\varepsilon}\right|^{2} d x \geq \kappa \pi .
$$

Proof. Assume that either (a) or (b) is violated for a sequence $\varepsilon_{k} \rightarrow 0$ and some $y=y_{k}$ such that $\rho_{\varepsilon_{k}}^{2}\left(y_{k}\right) \leq 1-\mu$. According to Lemma 22, $y_{k} \rightarrow \partial A$. For definiteness we suppose that $y_{k} \rightarrow \partial \Omega$. Then (by Lemma 22,

$$
\operatorname{dist}\left(y_{k}, \partial \Omega\right)=o\left(\varepsilon_{k}\right)
$$

Let $u_{\varepsilon}$ be extended in $\omega$ so that $\left\|u_{\varepsilon}\right\|_{H^{1}(\Omega)} \leq C\left\|u_{\varepsilon}\right\|_{H^{1}(A)}$ and $\left|u_{\varepsilon}\right| \leq 1$ in $\Omega$, where $C$ is independent of $\varepsilon$. Extend $h_{\varepsilon}(x)$ inside the domain $\omega$ by $h_{\varepsilon}=h_{\varepsilon}(\partial \omega)$. Following [8] we rescale $u_{\varepsilon_{k}}$ and $h_{\varepsilon_{k}}$ by a conformal map that "moves" $y_{k}$ away from the boundary. Fix a conformal mapping $\eta$ from $\Omega$ onto the unit disk $B_{1}(0)$. We introduce the conformal map $\zeta_{k}(z)=\left(z-\eta\left(y_{k}\right)\right) /\left(\bar{\eta}\left(y_{k}\right) z-1\right)$ from $B_{1}(0)$ onto itself and set $U_{k}(z)=$ $u_{\varepsilon_{k}}\left(\eta^{-1}\left(\zeta_{k}(z)\right)\right), H_{k}(z)=h_{\varepsilon_{k}}\left(\eta^{-1}\left(\zeta_{k}(z)\right)\right)$. It is easy to see that $\left\|U_{k}\right\|_{H^{1}\left(B_{1}(0)\right)} \leq C$ and $\left\|H_{k}\right\|_{H^{1}\left(B_{1}(0)\right)} \leq C$ with some $C$ independent of $k$. Therefore, without loss of generality, we can assume that $U_{k}$ and $H_{k}$ converge to limits $U$ and $H$ (respectively) weakly in $H^{1}$, as $k \rightarrow \infty$.

Arguing as in [8] (Section 4), we can show that $U_{k} \rightarrow U$ in $C_{\mathrm{loc}}^{1}\left(B_{1}(0)\right)$ and that $\Delta U=0$ in $B_{1}(0)$. Therefore, $|U(0)|^{2}=\lim _{k \rightarrow \infty}\left|U_{k}(0)\right|^{2}=\lim _{k \rightarrow \infty}\left|u_{\varepsilon_{k}}\left(y_{k}\right)\right|^{2} \leq$ $1-\mu$. We also have $|U|=1$ a.e. on $S^{1}$. We now show that $\partial_{z} U=0$ in $B_{1}(0)$. Indeed, by the maximum principle, $|U|<1$ in $B_{1}(0)$, hence $\max _{B_{t}(0)}\left|U_{k}(z)\right|^{2}<1-\varepsilon_{k}^{1 / 2}$ for any fixed $0<t<1$ and sufficiently large $k$. It follows that for such $k$ we have $\eta^{-1}\left(\zeta_{k}\left(B_{t}(0)\right)\right) \subset Q_{\varepsilon}^{+}$. Then, in view of 6.11 , we get, by using the conformality of the maps $\eta^{-1}$ and $\zeta_{k}$,

$$
\int_{B_{t}(0)}\left|\partial_{z} U_{k}\right|^{2} d x=\int_{\eta^{-1}\left(\zeta_{k}\left(B_{t}(0)\right)\right)}\left|\partial_{z} u_{\varepsilon_{k}}\right|^{2} d x \leq \int_{Q_{\varepsilon}^{+}}\left|\partial_{z} u_{\varepsilon_{k}}\right|^{2} d x \rightarrow 0 .
$$

This implies that $\partial_{z} U=0$ in $B_{1}(0)$.

In order to show (b), we use the pointwise equalities $\frac{1}{2}|\nabla U|^{2}=-\partial_{x_{1}} U \times \partial_{x_{2}} U+$ $2\left|\partial_{z} U\right|^{2}$ and $\partial_{z} U=0$ to obtain

$$
\frac{1}{2} \int_{B_{1}(0)}|\nabla U|^{2} d x=-\int_{B_{1}(0)} \partial_{x_{1}} U \times \partial_{x_{2}} U d x=-\pi \operatorname{deg}\left(U, S^{1}\right) .
$$

As $U \not \equiv$ const, we therefore have $\frac{1}{2} \int_{B_{1}(0)}|\nabla U|^{2} d x \geq \pi$. It follows that there is $0<$ $t<1$ such that

$$
\frac{1}{2} \int_{B_{t}(0)}|\nabla U|^{2} d x>\kappa \pi
$$

The image $\zeta_{k}\left(B_{t}(0)\right)$ of the disk $B_{t}(0)$ is the disk $B_{t_{k}}\left(\xi_{k}\right)$ with radius $t_{k}=\frac{t\left(1-\left|\eta\left(y_{k}\right)\right|^{2}\right)}{1-t^{2}\left|\eta\left(y_{k}\right)\right|^{2}}$ centered at $\xi_{k}=\frac{\eta\left(y_{k}\right)\left(1-t^{2}\right)}{1-t^{2}\left|\eta\left(y_{k}\right)\right|^{2}}$. According to $6.14, t_{k}=o\left(\varepsilon_{k}\right)$ for $k \rightarrow \infty$, hence 
$\eta^{-1}\left(B_{t_{k}}\left(\xi_{k}\right)\right) \subset B_{\varepsilon_{k}}\left(y_{k}\right) \cap A$ when $k$ is sufficiently large. Then, by using the conformal invariance and lower semicontinuity of the Dirichlet integral and bound (6.15), we get

$$
\begin{aligned}
\int_{B_{\varepsilon_{k}}\left(y_{k}\right) \cap A}\left|\nabla u_{\varepsilon_{k}}\right|^{2} d x & \geq \int_{\eta^{-1}\left(\zeta_{k}\left(B_{t}(0)\right)\right)}\left|\nabla u_{\varepsilon_{k}}\right|^{2} d x \\
& =\int_{B_{t}(0)}\left|\nabla U_{k}\right|^{2} d x>2 \kappa \pi \quad \text { as } k \rightarrow \infty .
\end{aligned}
$$

In order to show that $h_{\varepsilon_{k}}\left(y_{k}\right)=H_{k}(0)>1+\mu / 4$ when $k \rightarrow \infty$ we note that the system 2.1 is conformally invariant, i.e.

$$
\nabla^{\perp} H_{k}=\left(U_{k} \times \partial_{x_{1}} U_{k}, U_{k} \times \partial_{x_{2}} U_{k}\right) \quad \text { in } \zeta_{k}^{-1}(\eta(A)) .
$$

Then, bearing in mind the convergence properties of $U_{k}$, we find that $H_{k} \rightarrow H$ in $C_{\text {loc }}^{1}\left(B_{1}(0)\right)$ and

$$
\nabla^{\perp} H=\left(U \times \partial_{x_{1}} U, U \times \partial_{x_{2}} U\right)=-\frac{1}{2} \nabla^{\perp}\left(|U|^{2}\right) \quad \text { in } B_{1}(0),
$$

where we have used the fact that $\partial_{z} U=0$ in $B_{1}(0)$. Since $H=|U|=1$ on $\partial B_{1}(0)$ we have $H=\frac{3}{2}-\frac{1}{2}|U|^{2}$ in $B_{1}(0)$, therefore

$$
\lim _{k \rightarrow \infty} h_{\varepsilon_{k}}\left(y_{k}\right)=\lim _{k \rightarrow \infty} H_{k}(0)=\frac{3}{2}-\frac{1}{2}|U(0)|^{2} \geq 1+\mu / 2 .
$$

This completes the proof of the lemma.

Remark 24. Lemma 23 implies that in the case when $p=q=d$ minimizers of 1.7 ) are vortexless for sufficiently small $\varepsilon$. Indeed, by Theorem 4 they $H^{1}$-strongly converge, up to a subsequence, as $\varepsilon \rightarrow 0$ to a minimizing harmonic map $u \in \mathcal{J}_{d d}^{(d)}$. On the other hand, 6.13 exhibits the energy concentration property near zeros of minimizers, which is incompatible with the strong $H^{1}$-convergence.

The following result, which describes the structure of the function $h_{\varepsilon}$ for small $\varepsilon$, plays a crucial role in the proof of the main technical result (Lemma 27) in Section 7 .

Lemma 25. For sufficiently small $\varepsilon, \varepsilon<\varepsilon_{4}\left(\varepsilon_{4}>0\right)$, we have

(i) $\rho_{\varepsilon}^{2}(x) \geq 1 / 2$ when $h_{\varepsilon}(\partial \omega)-1 / 8 \leq h_{\varepsilon}(x) \leq 9 / 8$ and $h_{\varepsilon}(\partial \omega)<\min _{\mathcal{L}} h_{\varepsilon}(x) \leq$ $\max _{\mathcal{L}} h_{\varepsilon}(x)<h_{\varepsilon}(\partial \Omega)$; if $\rho_{\varepsilon}^{2}(x)<1 / 2$, then either

$$
\operatorname{dist}(x, \partial \Omega)<\operatorname{dist}(\mathcal{L}, \partial \Omega) \text { and } h_{\varepsilon}(x)>9 / 8 \text {, }
$$

or

$$
\operatorname{dist}(x, \partial \omega)<\operatorname{dist}(\mathcal{L}, \partial \omega) \quad \text { and } \quad h_{\varepsilon}(x)<h_{\varepsilon}(\partial \omega)-1 / 8
$$

(ii) there exist $x_{\varepsilon}^{*} \in \partial \Omega$ and $x_{\varepsilon}^{* *} \in \partial \omega$ such that $\frac{\partial h_{\varepsilon}}{\partial v}\left(x_{\varepsilon}^{*}\right)>0$ and $\frac{\partial h_{\varepsilon}}{\partial v}\left(x_{\varepsilon}^{* *}\right)>0$. 
Proof. (i) follows from Lemma 22, Lemma 23 and the convergence properties of $h_{\varepsilon}$ (as $\varepsilon \rightarrow 0$ ) established in the proof of Theorem 4. To prove (ii) we argue as follows. Let $\hat{\varepsilon}_{2}(\mu, \kappa)$ be the best (biggest) constant in Lemma 23 . Then $\hat{\varepsilon}_{2}(\mu, \kappa)$ is increasing in $\mu$ and decreasing in $\kappa$. For $j=2,3, \ldots$ set

$$
\hat{\mu}_{\varepsilon}=1 / j \quad \text { when } \min \left\{\hat{\varepsilon}_{2}\left(\frac{1}{j+1}, \frac{j}{j+1}\right), \frac{1}{j+1}\right\} \leq \varepsilon<\min \left\{\hat{\varepsilon}_{2}\left(\frac{1}{j}, \frac{j-1}{j}\right), \frac{1}{j}\right\}
$$

(this defines $\hat{\mu}_{\varepsilon}>0$ for all $0<\varepsilon<\min \left\{\hat{\varepsilon}_{2}(1 / 2,1 / 2), 1 / 2\right\}$ ). Then $\hat{\mu}_{\varepsilon} \rightarrow 0$ as $\varepsilon \rightarrow 0$ and 6.13) is satisfied with $\kappa=1-\hat{\mu}_{\varepsilon}$ when $\rho_{\varepsilon}^{2}(y)<1-\hat{\mu}_{\varepsilon}$; the same is true when $\hat{\mu}_{\varepsilon}$ is replaced with $\mu_{\varepsilon}=\max \left\{\hat{\mu}_{\varepsilon}, \varepsilon^{1 / 2}\right\}$. We pick a point $x_{\varepsilon}^{(1)}$ in $A$ such that $\rho_{\varepsilon}^{2}\left(x_{\varepsilon}^{(1)}\right)<1-\mu_{\varepsilon}$; then we pick a point $x_{\varepsilon}^{(2)}$ in $A \backslash B_{2 \varepsilon}\left(x_{\varepsilon}^{(1)}\right)$ such that $\rho_{\varepsilon}^{2}\left(x_{\varepsilon}^{(2)}\right)<1-\mu_{\varepsilon}$, etc., unless for some $K_{\varepsilon}$ we have $\rho_{\varepsilon}^{2}(x) \geq 1-\mu_{\varepsilon}$ on $A \backslash \bigcup_{k=1}^{K_{\varepsilon}} B_{2 \varepsilon}\left(x_{\varepsilon}^{(k)}\right)$. By the construction of $\mu_{\varepsilon}$, since the disks $B_{\varepsilon}\left(x_{\varepsilon}^{(k)}\right)$ are disjoint,

$$
\frac{1}{2} \int_{A}\left|\nabla u^{\varepsilon}\right|^{2} d x \geq \frac{1}{2} \sum_{1}^{K_{\varepsilon}} \int_{A \cap B_{\varepsilon}\left(x_{\varepsilon}^{(k)}\right)}\left|\nabla u^{\varepsilon}\right|^{2} d x \geq K_{\varepsilon}\left(1-\mu_{\varepsilon}\right) \pi .
$$

Therefore, by (6.1), we have a uniform bound $K_{\varepsilon} \leq C$. Arguing as in [11, Chapter IV, Theorem IV.1] we can increase the radii of disks to $\varepsilon \lambda>2 \varepsilon$ (with $\lambda$ independent of $\varepsilon$ ) and take a subset $I_{\varepsilon}$ of $\left\{1, \ldots, K_{\varepsilon}\right\}$ such that

$$
\bigcup_{k \in I_{\varepsilon}} B_{\varepsilon \lambda}\left(x_{\varepsilon}^{k}\right) \supset \bigcup_{k=1}^{K_{\varepsilon}} B_{2 \varepsilon}\left(x_{\varepsilon}^{k}\right) \quad \text { and } \quad \operatorname{dist}\left(x_{\varepsilon}^{k^{\prime}}, x_{\varepsilon}^{k}\right)>4 \varepsilon \lambda \quad \text { for different } k, k^{\prime} \in I_{\varepsilon} .
$$

We also have

$$
\rho_{\varepsilon}^{2}(x) \geq 1-\mu_{\varepsilon} \quad \text { in } A \backslash \bigcup_{k \in I_{\varepsilon}} B_{\varepsilon \lambda}\left(x_{\varepsilon}^{k}\right)
$$

Assume $h_{\varepsilon}$ and $h_{0}$ are extended to the entire $\mathbb{R}^{2}$ by $h_{\varepsilon}=h_{\varepsilon}(\partial \omega), h_{0}=h_{0}(\partial \omega)$ in $\omega$, and $h_{\varepsilon}=h_{0}=1$ in $\mathbb{R}^{2} \backslash \Omega$. Since $\rho_{\varepsilon}^{2}(x) \geq 1-\mu_{\varepsilon} \geq 1-\varepsilon^{1 / 2}$ in $D_{\varepsilon}^{(k)}=$ $B_{2 \lambda \varepsilon}\left(x_{\varepsilon}^{(k)}\right) \backslash B_{\lambda \varepsilon}\left(x_{\varepsilon}^{(k)}\right)$, by 6.10 we have

$$
\int_{D_{\varepsilon}^{(k)}}\left|\nabla h_{\varepsilon}\right|^{2} d x \leq 2 \int_{D_{\varepsilon}^{(k)}}\left(\left|\nabla\left(h_{\varepsilon}-h_{0}\right)\right|^{2}+\left|\nabla h_{0}\right|^{2}\right) d x \rightarrow 0 \quad \text { as } \varepsilon \rightarrow 0 .
$$

Then, writing the integral over $D_{\varepsilon}^{(k)}$ in polar coordinates centered at $x_{\varepsilon}^{(k)}$, and using Fubini's theorem, we can find $\lambda_{\varepsilon}^{(k)}, \lambda \varepsilon \leq \lambda_{\varepsilon}^{(k)} \leq 2 \lambda \varepsilon$, such that

$$
\int_{\left|x-x_{\varepsilon}^{(k)}\right|=\lambda_{\varepsilon}^{(k)}}\left|\nabla h_{\varepsilon}\right|^{2} d \sigma=o(1 / \varepsilon) .
$$

Therefore, by the Cauchy-Schwarz inequality,

$$
\int_{\left|x-x_{\varepsilon}^{(k)}\right|=\lambda_{\varepsilon}^{(k)}}\left|\nabla h_{\varepsilon}\right| d s \leq\left(2 \pi \lambda_{\varepsilon}^{(k)}\right)^{1 / 2}\left\{\int_{\left|x-x_{\varepsilon}^{(k)}\right|=\lambda_{\varepsilon}^{(k)}}\left|\nabla h_{\varepsilon}\right|^{2} d s\right\}^{1 / 2}=o(1)
$$


We now integrate 2.4 over $Q^{+} \backslash \bigcup_{k \in I_{\varepsilon}} B_{\lambda_{\varepsilon}^{(k)}}\left(x_{\varepsilon}^{(k)}\right)$ using 6.16 and Lemma 13 to obtain

$$
\int_{\Gamma_{\varepsilon}} \frac{\partial h_{\varepsilon}}{\partial v} d s=2 \pi d+\sum_{k \in I_{\varepsilon}^{\prime}} \int_{\left|x-x_{\varepsilon}^{(k)}\right|=\lambda_{\varepsilon}^{(k)}} \frac{\partial h_{\varepsilon}}{\partial v} \frac{d s}{\rho_{\varepsilon}^{2}}=2 \pi d-o(1) \quad \text { when } \varepsilon \rightarrow 0 .
$$

Here $I_{\varepsilon}^{\prime}$ denotes the subset of indices $k \in I_{\varepsilon}$ such that $B_{\lambda_{\varepsilon}^{(k)}}\left(x_{\varepsilon}^{(k)}\right) \cap Q^{+} \neq \emptyset$, and $\Gamma_{\varepsilon}=$ $\partial \Omega \backslash \bigcup_{k \in I_{\varepsilon}^{\prime}} B_{\lambda_{\varepsilon}^{(k)}}\left(x_{\varepsilon}^{(k)}\right)$. Therefore, there is $x_{\varepsilon}^{*} \in \Gamma_{\varepsilon}$ such that $\frac{\partial h_{\varepsilon}}{\partial v}\left(x_{\varepsilon}^{*}\right)>0$. Similarly, we can show that on $\gamma_{\varepsilon}=\partial \omega \backslash \bigcup_{k \in I_{\varepsilon} \backslash I_{\varepsilon}^{\prime}} B_{\lambda_{\varepsilon}^{(k)}}\left(x_{\varepsilon}^{(k)}\right)$ there is $x_{\varepsilon}^{* *}$ such that $\frac{\partial h_{\varepsilon}}{\partial \nu}\left(x_{\varepsilon}^{* *}\right)>0$.

\section{Inductive proof of Theorem 3}

Fix $\Lambda>0$ and an integer $d>0$ such that $\Lambda>I_{0}(d, A)$, and let $\mathfrak{x}_{0}$ be the greatest integer such that

$$
I_{0}(d, A)+\pi \mathfrak{x}_{0}<\Lambda .
$$

Clearly, $\mathfrak{x}_{0} \geq 0$. In this section we show that, for small $\varepsilon$, the infimum in problem (1.7) is always attained, provided the integers $p, q$ satisfy

$$
\mathfrak{x}(p, q) \leq \mathfrak{x}_{0} \quad \text { and } \quad p \leq d, q \leq d,
$$

where $æ(p, q)=|d-q|+|d-p|$.

Proposition 26. Given an integer $K \leq \mathfrak{x}_{0}$, let $p \leq d, q \leq d$ be integers such that $\mathfrak{x}(p, q) \leq K$. Then, for sufficiently small $\varepsilon$, the infimum in problem (1.7) is always attained and

$m_{\varepsilon}(p, q, d) \leq m_{\varepsilon}(l, m, d)+\pi((l-p)+(m-q)) \quad$ when $p \leq l \leq d, q \leq m \leq d$.

Moreover, the inequality in (7.2) is strict unless $l=p$ and $m=q$.

Proof. The proof is by induction in $K$. The basis of induction $(K=0)$ is established in Section 5 (cf. Lemma 19). The demonstration of the induction step relies on the following lemma, whose proof is at the end of this section.

Lemma 27. Assume the integers $p$ and $q$ satisfy (7.1), and for $\varepsilon<\varepsilon_{5}, \varepsilon_{5}>0$, there exists a minimizer $u_{\varepsilon}$ of problem (1.7) whose GL energy $E_{\varepsilon}\left(u_{\varepsilon}\right)$ satisfies (6.1). Then for any $\varepsilon<\min \left\{\varepsilon_{4}, \varepsilon_{5}\right\}$ (where $\varepsilon_{4}$ is as in Lemma 25) there exists $v_{\varepsilon} \in \mathcal{J}_{p(q-1)}$ such that $\operatorname{abdeg}\left(v_{\varepsilon}\right) \in(d-1 / 2, d+1 / 2)$ and

$$
E_{\varepsilon}\left(v_{\varepsilon}\right)<m_{\varepsilon}(p, q, d)+\pi .
$$

Similarly, in $\mathcal{J}_{(p-1) q}$ there exists a testing map (still denoted $v_{\varepsilon}$ ) satisfying 7.3 and such that abdeg $\left(v_{\varepsilon}\right) \in(d-1 / 2, d+1 / 2)$. 
In view of Lemma 27, to prove the claim of Proposition 26 for $K+1$ in place of $K$, it suffices to show that $m_{\varepsilon}(p, q-1, d)$ is always attained for sufficiently small $\varepsilon$ whenever $p \leq d, q \leq d$ and $æ(p, q)=K$ (the attainability of $m_{\varepsilon}(p-1, q, d)$ is proved analogously). Let $u$ be a weak $H^{1}$-limit of the minimizing sequence $\left\{u^{(k)}\right\} \subset \mathcal{J}_{p(q-1)}^{(d)}$. According to Lemma 27, such a minimizing sequence exists. Moreover, this lemma, with the induction hypothesis, implies

$$
\limsup _{k \rightarrow \infty} E_{\varepsilon}\left(u^{(k)}\right)<m_{\varepsilon}\left(l^{\prime}, m^{\prime}, d\right)+\pi\left(l^{\prime}-p\right)+\pi\left(m^{\prime}-(q-1)\right)
$$

for any integers $l^{\prime}, m^{\prime}$ satisfying $p \leq l^{\prime} \leq d, q-1 \leq m^{\prime} \leq d$ and $æ\left(l^{\prime}, m^{\prime}\right) \leq K$. We know that $\operatorname{abdeg}(u)=\lim _{k \rightarrow \infty} \operatorname{abdeg}\left(u^{(k)}\right) \in(d-1 / 2, d+1 / 2)$ when $\varepsilon<\varepsilon_{0}$ (where $\varepsilon_{0}=$ $\varepsilon_{0}(\Lambda)$ is as in Proposition 2), hence $u$ is a solution of the GL equation (1.1) (see arguments in Lemma 19. Therefore, if we write $\liminf _{k \rightarrow \infty} E_{\varepsilon}\left(u^{(k)}\right) \leq \limsup _{k \rightarrow \infty} E_{\varepsilon}\left(u^{(k)}\right)$ and apply Lemma 9 and Lemma 18 successively to the left hand side, we find, using (7.4) with $l^{\prime}=m^{\prime}=d$, that for sufficiently small $\varepsilon$,

$I_{0}(d, A)-\frac{\pi}{2}+\pi æ(l, m)+\pi(|l-p|+|m-(q-1)|) \leq m_{\varepsilon}(d, d, d)+\pi æ(p, q-1)$,

where $l=\operatorname{deg}(u, \partial \omega), m=\operatorname{deg}(u, \partial \Omega)$. Thanks to Lemma $16 m_{\varepsilon}(d, d, d) \leq I_{0}(d, A)$. Thus

$|l-d|+|l-p| \leq|p-d|+1 / 2$ and $|m-d|+|m-(q-1)| \leq|(q-1)-d|+1 / 2$.

Since $l$ and $m$ are integers, it follows that $p \leq l \leq d$ and $q-1 \leq m \leq d$. Now, assuming $l \neq p$ or $m \neq q-1$, we use Lemma 9 and $\overline{7.4}$ with $l^{\prime}=l, m^{\prime}=m$ to obtain

$E_{\varepsilon}(u)+\pi(l-p+m-(q-1)) \leq \liminf _{k \rightarrow \infty} E_{\varepsilon}\left(u^{(k)}\right)<m_{\varepsilon}(l, m, d)+\pi(l-p+m-(q-1))$,

i.e. $E_{\varepsilon}(u)<m_{\varepsilon}(l, m, d)$. On the other hand, $u \in \mathcal{J}_{l m}^{(d)}$, implying that $E_{\varepsilon}(u) \geq m_{\varepsilon}(l, m, d)$ : this is a contradiction. Therefore, $l=p$ and $m=q-1$; i.e., $u$ is an admissible testing map in problem (1.7) and thus the infimum $m_{\varepsilon}(p, q-1, d)$ is always attained.

Proof of Lemma 27. For simplicity we drop the subscript $\varepsilon$. The underlying idea is to modify the minimizer $u$ of $(1.7)$ in a neighborhood of $\partial \Omega$ as in Proposition 20 (see Section 5). In general, $u$ may have zeros now. Thus, the arguments need to be more delicate. Loosely speaking, we construct a testing map $v$ with an additional "vortex" located "near" $x^{*}$, where $x^{*}$ is a point on $\partial \Omega$ such that $\frac{\partial h}{\partial v}\left(x^{*}\right)>0$ (cf. Lemma 25 .

Step 1: Domain decomposition. Let $1-\delta$, where $\delta>0$, be a regular value of $h$ (thanks to Sard's lemma this holds for almost all $\delta$ ). Consider the subdomain of $A$ where $h>1-\delta$. There is a (unique) connected component $D_{\delta}$ of this subdomain such that $\partial D_{\delta} \supset \partial \Omega$. Since $h(\partial \Omega)=1>h(\partial \omega)$, when $\delta$ is sufficiently small, the boundary of $D_{\delta}$ contains a connected component $\Gamma_{\delta} \neq \partial \Omega$ enclosing $\omega$. According to Lemma 25, if we choose $\delta<\delta_{0}\left(\delta_{0}>0\right)$, then the domain enclosed by $\Gamma_{\delta}$ and $\partial \Omega$ will lie away from the contour $\mathcal{L}$; i.e. $\Gamma_{\delta}$ also encloses $\mathcal{L}$. Moreover, if $\rho(x)(=|u(x)|)$ vanishes at a point $x$ in this domain, then $h(x)>1$. Therefore, the minimum of $h$ over the closure of the aforementioned 
domain cannot be attained at any interior point; otherwise, $h$ satisfies $\operatorname{div}\left(\rho^{-2} \nabla h\right)=0$ in a neighborhood of that point, which is impossible. In other words, $h>1-\delta$ in the domain enclosed by $\Gamma_{\delta}$ and $\partial \Omega$; i.e., this domain coincides with $D_{\delta}$. Thus, the boundary of $D_{\delta}$ consists of exactly the two connected components $\partial \Omega$ and $\Gamma_{\delta}$. Also, possibly for a smaller $\delta_{0}$, the set $P=\left\{x \in D_{\delta} ; h(x) \geq 1\right\}$ is independent of $\delta$ (recall that $\delta<\delta_{0}$ and $1-\delta$ is a regular value of $h)$. Indeed, consider the set $S_{\delta}=\{x \in A ; h(x)>\alpha\} \cap D_{\delta}$ $\left(0<\delta<\delta_{0}\right)$, where $\alpha$ is a regular value of $h$ and $1<\alpha<9 / 8$. The set $S_{\delta}$ consists of a finite number $n(\delta)$ of connected components. Since $D_{\delta} \supset D_{\delta^{\prime}}$ for $\delta>\delta^{\prime}$, the function $n(\delta)$ is nondecreasing, hence $n(\delta)=\lim _{\delta^{\prime} \rightarrow 0} n\left(\delta^{\prime}\right)$ when $0<\delta<\delta_{0}$ (for some $\delta_{0}>0$ ) and $S_{\delta}=S_{\delta^{\prime}}$ for $\delta, \delta^{\prime} \in\left(0, \delta_{0}\right)$. It follows that $1-\delta<h<\alpha$ in $D_{\delta} \backslash D_{\delta^{\prime}}$ when $0<\delta^{\prime}<\delta<\delta_{0}$. For such $\delta$ and $\delta^{\prime}$ the function $h$ satisfies $\operatorname{div}\left(\rho^{-2} \nabla h\right)=0$ in $D_{\delta} \backslash D_{\delta^{\prime}}$ (by Lemma 25, while $h<1$ on the boundary of $D_{\delta} \backslash D_{\delta^{\prime}}$. Thus $h<1$ in $D_{\delta} \backslash D_{\delta^{\prime}}$. We now see that $\left\{x \in D_{\delta} ; h(x) \geq 1\right\}=P:=\bigcap_{\delta^{\prime}<\delta_{0}} D_{\delta^{\prime}}$, when $0<\delta<\delta_{0}$, as required.

Thus we have, for $\delta<\delta_{0}$,

$$
d^{\prime}:=\frac{1}{2 \pi} \int_{\Gamma_{\delta}} \frac{\partial h}{\partial v} \frac{d s}{\rho^{2}}=\frac{1}{2 \pi} \int_{\Gamma_{\delta}} \frac{u}{|u|} \times \frac{\partial}{\partial \tau} \frac{u}{|u|} d s=\operatorname{deg}\left(u, \Gamma_{\delta}\right)>0
$$

$\left(1-\delta=h\left(\Gamma_{\delta}\right)\right.$ is a regular value of $h$ and $h>1-\delta$ in $D_{\delta}$, while $h$ satisfies $\operatorname{div}\left(\rho^{-2} \nabla h\right)$ $=0$ in $D_{\delta} \backslash P$, hence $\partial h / \partial v>0$ on $\left.\Gamma_{\delta}\right)$ and the integer $d^{\prime}$ is independent of $\delta$. Therefore $u$ admits the representation $u=\rho e^{i d^{\prime} \theta}$ in $G_{\delta}=D_{\delta} \backslash P$, where $\theta: G_{\delta} \rightarrow \mathbb{R} / 2 \pi \mathbb{Z}$ is a smooth function and $\rho>0$ in $\bar{G}_{\delta}$. Moreover, for every $0<\delta^{\prime} \leq \delta$ such that $1-\delta^{\prime}$ is a regular value of $h$ we have $\partial \theta / \partial \tau>0$ on $\Gamma_{\delta^{\prime}}\left(\nabla h=-d^{\prime} \rho^{2} \nabla^{\perp} \theta\right.$ and $\partial h / \partial v>0$ on $\left.\Gamma_{\delta^{\prime}}\right)$ and the total variation $\mathrm{V}\left(\theta, \Gamma_{\delta^{\prime}}\right)$ of $\theta$ on $\Gamma_{\delta^{\prime}}$ is $\mathrm{V}\left(\theta, \Gamma_{\delta^{\prime}}\right)=2 \pi \operatorname{deg}\left(e^{i \theta}, \Gamma_{\delta^{\prime}}\right)=2 \pi$.

Without loss of generality, we can assume that $u\left(x^{*}\right)=1$. Since $\frac{\partial h}{\partial v}\left(x^{*}\right)>0$, we have $|\nabla h|>0$ in $A \cap G^{\prime}$ for some neighborhood $G^{\prime}$ of $x^{*}$. We also have $\rho>0$ in $\overline{A \cap G^{\prime}}$ (possibly by choosing a smaller $G^{\prime}$ ). Then, since $\nabla h=-d^{\prime} \rho^{2} \nabla^{\perp} \theta$, the map $x \mapsto(h, \theta)$ is a $C^{1}$-diffeomorphism from $A \cap G^{\prime}$ to its image. Clearly, we can assume that $A \cap G^{\prime}$ is a simply connected domain hence we can fix a single-valued branch of $\theta$ in $A \cap G^{\prime}$ such that $\theta\left(x^{*}\right)=0$. Set $G_{\delta}^{\prime}:=\left\{x \in A \cap G^{\prime} ; 1-\delta<h<1, \theta \in(-\delta, \delta)\right\}$. By choosing $\delta<\delta_{0}$ small enough we have $\overline{G_{\delta}^{\prime}} \subset G^{\prime}$. We can also assume, without loss of generality, that $1-\delta$ is a regular value of $h$, since by Sard's lemma $1-\delta$ is a regular value of $h$ for almost all $\delta$.

Next, we show that $\theta(x)$ does not take values from $(-\delta, \delta)+2 \pi \mathbb{Z}$ in $G_{\delta} \backslash \overline{G_{\delta}^{\prime}}$. Indeed, choose some $\delta^{\prime} \in(0, \delta)$ such that $1-\delta^{\prime}$ is a regular value of $h$, and consider the simply connected domain $\left\{x \in G_{\delta} \backslash \overline{G_{\delta}^{\prime}} ; 1-\delta<h(x)<1-\delta^{\prime}\right\}$. Its boundary consists of the segments $\Gamma_{\delta}^{\prime}=\Gamma_{\delta} \backslash\left(\Gamma_{\delta} \cap \overline{G_{\delta}^{\prime}}\right)$ and $\Gamma_{\delta^{\prime}}^{\prime}=\Gamma_{\delta^{\prime}} \backslash\left(\Gamma_{\delta^{\prime}} \cap \overline{G_{\delta}^{\prime}}\right)$, and the shortest curves (where $\theta= \pm \delta(\bmod 2 \pi \mathbb{Z}))$ connecting $\Gamma_{\delta}^{\prime}$ and $\Gamma_{\delta^{\prime}}^{\prime}$ along opposite faces of $G_{\delta}^{\prime}$. There is a singlevalued branch of $\theta$ in this domain such that $\delta \leq \theta \leq 2 \pi-\delta$ on its boundary $(\partial \theta / \partial \tau>0$ on $\Gamma_{\delta}$ and $\Gamma_{\delta^{\prime}}$, and $\left.\mathrm{V}\left(\theta, \Gamma_{\delta}\right)=\mathrm{V}\left(\theta, \Gamma_{\delta^{\prime}}\right)=2 \pi\right)$. Since $\theta$ satisfies $\operatorname{div}\left(\rho^{2} \nabla \theta\right)=0$ we have $\delta \leq \theta \leq 2 \pi-\delta$ everywhere in the domain by the maximum principle. By assuming $\delta^{\prime} \rightarrow 0$ we get $\delta \leq \theta \leq 2 \pi-\delta$ in $G_{\delta} \backslash \overline{G_{\delta}^{\prime}}$. Therefore, $G_{\delta}^{\prime}$ can be equivalently described 


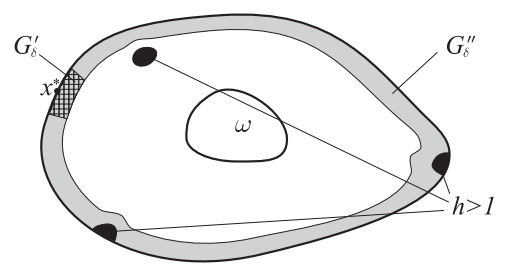

Fig. 1. Domain decomposition.

as

$$
x \in G_{\delta}^{\prime} \Leftrightarrow x \in G_{\delta}, 1-\delta<h(x)<1, \theta(x) \in(-\delta, \delta)+2 \pi \mathbb{Z} .
$$

Thus we have $A=G_{\delta}^{\prime} \cup G_{\delta}^{\prime \prime} \cup\left(A \backslash G_{\delta}\right)$ (see Fig. 1 , where $G_{\delta}^{\prime \prime}=G_{\delta} \backslash G_{\delta}^{\prime}$.

Step 2: Construction of the testing map. We construct the testing map $v$, which will have the form

$$
v= \begin{cases}u & \text { in } A \backslash G_{\delta}, \\ \rho w_{t} & \text { in } G_{\delta}\end{cases}
$$

with $w_{t}=w_{t}(h(x), \theta(x))$ is unknown for the moment. We impose the following boundary conditions on $\partial G_{\delta}$ :

$$
\begin{gathered}
w_{t}=e^{i d^{\prime} \theta} \frac{e^{-i \theta}-(1-t \varphi(\theta))}{e^{-i \theta}(1-t \varphi(\theta))-1} \quad \text { on } \partial G_{\delta} \backslash \Gamma_{\delta}, \\
w_{t}=e^{i d^{\prime} \theta} \quad \text { on } \Gamma_{\delta},
\end{gathered}
$$

where $0 \leq \varphi \leq 1$ is a smooth $2 \pi$-periodic cut-off function such that $\varphi(\theta)=1$ when $\theta \in(-\delta / 2, \delta / 2)+2 \pi \mathbb{Z}$ and $\varphi(\theta)=0$ if $\theta \notin(-\delta, \delta)+2 \pi \mathbb{Z}$. Let $w_{t}=w_{t}(h, \theta)$ be a smooth $2 \pi$-periodic (in $\theta$ ) map, defined in the strip $1-\delta \leq h \leq 1$, which satisfies (7.7) and (7.8), when $h=1$ and $h=1-\delta$, respectively. Then 7.6 defines a family of maps $v \in H^{1}\left(A ; \mathbb{R}^{2}\right)$ for $0<t<1$ such that $|v|=1$ on $\partial A$ and

$$
\operatorname{deg}(v, \partial \Omega)=q-1, \quad \operatorname{deg}(v, \partial \omega)=p .
$$

We expand the right hand side of 7.7 into a series to get

$$
e^{i d^{\prime} \theta} \frac{e^{-i \theta}-(1-t \varphi(\theta))}{e^{-i \theta}(1-t \varphi(\theta))-1}=\left(1-t b_{-1}(t)\right) e^{i d^{\prime} \theta}+t \sum_{k \neq-1} b_{k}(t) e^{-i\left(k-d^{\prime}+1\right) \theta} .
$$

We next set

$$
w_{t}(h, \theta)=\left(1-t b_{-1}(t) f_{-1}(h)\right) e^{i d^{\prime} \theta}+t \sum_{k \neq-1} b_{k}(t) f_{k}(h) e^{-i\left(k-d^{\prime}+1\right) \theta},
$$


where the functions $f_{k}$ are defined by 5.20 with $k_{ \pm}= \pm\left(1 / d^{\prime}\right) \sqrt{\left(k-d^{\prime}+1\right)^{2}+\lambda-d^{\prime 2}}$. The positive parameters $t<1$ and $\lambda \geq 2 d^{\prime 2}$ are to be specified below. The coefficients $b_{k}(t)$ have the following property:

$$
\left|b_{k}(t)-c_{k}(t)\right| \leq C(1+|k|)^{-n}, \quad \forall n>0,
$$

where $C=C(n)$ is independent of $t, c_{k}=(t-2)(1-t)^{k}$ for $k \geq 0, c_{-1}=1$, and $c_{k}=0$ for $k<-1$. The estimate (7.12) is obtained, in a standard way, by comparing the Fourier coefficients in 7.10 with those of $e^{i d^{\prime} \theta}\left(e^{-i \theta}-(1-t)\right) /\left(e^{-i \theta}(1-t)-1\right)$.

Step 3: Verification of 7.3 . Thanks to Lemma 21, we have (since $\left|w_{t}\right|=1$ on $\partial G_{\delta}$ due to 7.7 7.8)

$$
E_{\varepsilon}(v)=E_{\varepsilon}(u)+L_{\varepsilon}^{\left(d^{\prime}\right)}\left(w_{t}, G_{\delta}\right)
$$

where the functional $L_{\varepsilon}^{\left(d^{\prime}\right)}(w)$ is defined as in 5.8. We now show that, for sufficiently small $t$,

$$
L_{\varepsilon}^{\left(d^{\prime}\right)}\left(w_{t}, G_{\delta}\right)<\pi
$$

To this end, as in the proof of Proposition 20, we consider the quadratic functional

$$
M_{\lambda}^{\prime}\left(w_{t}\right)=\frac{1}{2} \int_{G_{\delta}}\left(d^{\prime 2}\left|\partial_{h} w_{t}\right|^{2}+\left|\partial_{\theta} w_{t}\right|^{2}+\lambda\left|w_{t}-e^{i \theta}\right|^{2}-d^{\prime 2}\left|w_{t}\right|^{2}\right) \rho^{2}|\nabla \theta|^{2} d x .
$$

(One can actually show that $w_{t}$ minimizes 7.14 under the boundary conditions 7.7 7.8.). Since $\nabla h=-d^{\prime} \rho^{2} \nabla^{\perp} \theta$ in $G_{\delta}$ and $\rho \leq 1$ in $A$, we have

$$
\int_{G_{\delta}} \rho^{2}\left|\nabla w_{t}\right|^{2} d x \leq \int_{G_{\delta}}\left(d^{\prime 2}\left|\partial_{h} w_{t}\right|^{2}+\left|\partial_{\theta} w_{t}\right|^{2}\right) \rho^{2}|\nabla \theta|^{2} d x .
$$

Moreover, if we put

$$
\lambda=\max \left\{\frac{9}{2 \varepsilon^{2} \min \overline{G_{\delta}^{\prime}}|\nabla \theta|^{2}}, 2 d^{\prime 2}\right\}
$$

then, under the additional assumption that $\left|w_{t}\right| \leq 2$ in $G_{\delta}^{\prime}$, the pointwise inequality $2 \varepsilon^{2} \lambda\left|w_{t}-e^{i \theta}\right|^{2}|\nabla \theta|^{2} \geq \rho^{2}\left(\left|w_{t}\right|^{2}-1\right)^{2}$ holds in $G_{\delta}^{\prime}$ (see the proof of Proposition 20 . Thus,

$$
L_{\varepsilon}^{\left(d^{\prime}\right)}\left(w_{t}, G_{\delta}\right) \leq M_{\lambda}^{\prime}\left(w_{t}\right)+\frac{1}{4 \varepsilon^{2}} \int_{G_{\delta}^{\prime \prime}} \rho^{4}\left(\left|w_{t}\right|^{2}-1\right)^{2} d x .
$$

To obtain 7.13 we first note that

$$
M_{\lambda}^{\prime}\left(w_{t}\right)=\frac{t^{2} \pi}{d^{\prime}} \sum_{k=-\infty}^{\infty}\left|b_{k}(t)\right|^{2} \Phi_{k}^{\prime}\left(f_{k}\right),
$$

where the functional $\Phi_{k}^{\prime}$ is defined just as $\Phi_{k}$ in 5.19 with $d^{\prime}$ in place of $d$. While the representation (7.17) is analogous to (5.18), its justification is different because $\nabla h$ vanishes at least at some points of the boundary of $G_{\delta}$ (and possibly somewhere in $G_{\delta}^{\prime \prime}$ ). We rely on the following lemma, which directly implies 7.17). 
Lemma 28. Let $f, g \in C^{1}([1-\delta, 1] ; \mathbb{C})$. Then for all integers $n, m$,

$$
\int_{G_{\delta}} f(h) e^{i n \theta} \overline{g(h) e^{i m \theta}} \rho^{2}|\nabla \theta|^{2} d x= \begin{cases}0 & \text { if } n \neq m, \\ \frac{2 \pi}{d^{\prime}} \int_{1-\delta}^{1} f(s) \bar{g}(s) d s & \text { if } n=m .\end{cases}
$$

Proof. By virtue of the pointwise equalities $\nabla h \cdot \nabla \theta=0$ and $\operatorname{div}\left(\rho^{2} \nabla \theta\right)=0$ in $G_{\delta}$, for any regular values $\alpha, \beta$ of $h$ such that $1-\delta \leq \alpha<\beta \leq 1$ and any integer $n \neq m$,

$$
\begin{aligned}
\int_{\alpha<h<\beta} f e^{i n \theta} \overline{g e^{i m \theta}} \rho^{2}|\nabla \theta|^{2} d x=\frac{-i}{n-m} \int_{\alpha<h<\beta} \nabla \theta \cdot \nabla e^{i(n-m) \theta} f \bar{g} \rho^{2} d x \\
=\frac{i}{n-m} \int_{\alpha<h<\beta} \operatorname{div}\left(\rho^{2} \nabla \theta\right) f \bar{g} e^{i(n-m) \theta} d x \\
\quad+\frac{i}{n-m} \int_{\alpha<h<\beta} \nabla \theta \cdot \nabla h\left(f^{\prime} \bar{g}+f \bar{g}^{\prime}\right) e^{i(n-m) \theta} \rho^{2} d x=0,
\end{aligned}
$$

where all the integrals are over subsets of $G_{\delta}$. If $n=m$ we set $F(h)=\int_{\alpha}^{r} f(s) \bar{g}(s) d s$. Then, since $|\nabla h|=d^{\prime} \rho^{2}|\nabla \theta|$, we have

$$
\begin{aligned}
\int_{\alpha<h<\beta} f(h) \bar{g}(h) \rho^{2}|\nabla \theta|^{2} d x=\frac{1}{d^{\prime 2}} \int_{\alpha<h<\beta} \nabla(F(h)) \cdot \nabla h \frac{d x}{\rho^{2}} \\
=\frac{1}{d^{\prime 2}} F(\beta) \int_{h=\beta} \frac{\partial h}{\partial v} \frac{d s}{\rho^{2}}-\frac{1}{d^{\prime 2}} \int_{\alpha<h<\beta} \operatorname{div}\left(\frac{1}{\rho^{2}} \nabla h\right) F(h) d x \\
=\frac{2 \pi}{d^{\prime}} \int_{\alpha}^{\beta} f(s) \bar{g}(s) d s .
\end{aligned}
$$

Here we have also used the fact that $\operatorname{div}\left(\rho^{-2} \nabla h\right)=0$ in $G_{\delta}$. The statement of the lemma is then obtained by passing to the limits $\alpha \rightarrow 1-\delta$ and $\beta \rightarrow 1$ in 7.18,7.19.

Using 7.12 in 7.17, we compute

$$
\begin{array}{r}
M_{\lambda}^{\prime}\left(w_{t}\right)=\frac{t^{2} \pi}{d^{\prime}} \sum_{k=-\infty}^{\infty}\left|c_{k}(t)\right|^{2} \Phi_{k}^{\prime}\left(f_{k}\right)+O\left(t^{2}\right)=\pi\left((1-t)^{2}-1\right)^{2} \sum_{k=0}^{\infty} k(1-t)^{2 k} \\
\quad+2 \pi t^{2}\left(\lambda-d^{\prime 2}\right) \sum_{k=1}^{\infty} \frac{(1-t)^{2 k}}{k}+O\left(t^{2}\right)=\pi-2 \pi t+o(t) .
\end{array}
$$

Simple but tedious computations also show that

$$
\begin{aligned}
w_{t} & =e^{i d^{\prime} \theta} \\
& +t(t-2) e^{\left(1-d^{\prime}\right)\left((h-1) / d^{\prime}-i \theta\right)} \sum_{k=1}^{\infty}\left((1-t) e^{(h-1) / d^{\prime}-i \theta}\right)^{k}\left(1+\frac{\lambda-\left(d^{\prime}\right)^{2}}{2 k d^{\prime}}(h-1)\right)+O(t) \\
& =e^{i d^{\prime} \theta}+t(t-2) e^{\left(1-d^{\prime}\right)\left((h-1) / d^{\prime}-i \theta\right)} /\left(1-(1-t) e^{(h-1) / d^{\prime}-i \theta}\right)+O(t),
\end{aligned}
$$


uniformly in $\theta$ and $h \in(1-\delta, 1)$. Here we have used (7.11), 7.12) and the explicit form of the functions $f_{k}$. This yields

$$
\left|w_{t}-e^{i d^{\prime} \theta}\right| \leq C t \quad \text { when } \theta \notin(-\alpha, \alpha)+2 \pi \mathbb{Z},
$$

for any $0<\alpha<2 \pi$, where $C$ is independent of $t$. From 7.21, we see that the second term in 7.16 is of order $O\left(t^{2}\right)$ as $t \rightarrow 0$. Combined with 7.20 this proves 7.13.

Final step. The bound (7.16) is established under the assumption that $\left|w_{t}\right| \leq 2$ in $G_{\delta}^{\prime \prime}$. Note that this inequality can always be achieved by replacing $w_{t}$ with $\tilde{w}_{t}:=$ $w_{t} \min \left\{1,2 /\left|w_{t}\right|\right\}$. This change increases neither the first term in (7.16) nor the second one. Thus, in order to complete the proof of the lemma, we need to show only that the map $v$ defined by (7.6) satisfies $d-1 / 2 \leq \operatorname{abdeg}(v) \leq d+1 / 2$ when $t$ is chosen sufficiently small. Indeed, due to $7.21,, w_{t}$ weakly $H^{1}$-converges to $e^{i d^{\prime} \theta}$. Therefore the norm $\|u-v\|_{L^{2}(A)}$ tends to 0 when $t \rightarrow 0$. Then, according to Lemma 10 , for small $t, \operatorname{abdeg}(v)$ is close to $\operatorname{abdeg}(u)$, while $d-1 / 2<\operatorname{abdeg}(u)<d+1 / 2$ and we are done.

Acknowledgments. Part of this work was done when V. Rybalko was visiting Penn State University. He is grateful for the hospitality and support of his visit. He also acknowledges the support of the Akhiezer fund. The work of L. Berlyand is partially supported by NSF grant DMS-0708324. The authors thank O. Misiats for his careful reading of the manuscript and suggestions. The authors are grateful to the referee for comments and suggestions which led to an improvement of the manuscript.

\section{References}

[1] Ahlfors, L. V.: Complex Analysis. 2nd ed., McGraw-Hill (1966) Zbl 0154.31904 MR 0188405

[2] Alama, S., Bronsard, L.: Vortices and pinning effects for the Ginzburg-Landau model in multiply connected domains. Comm. Pure Appl. Math. 59, 36-70 (2006) Zbl 1084.82021 MR 2180083

[3] Almeida, L.: Topological sectors for Ginzburg-Landau energies. Rev. Mat. Iberoamer. 15, 487-545 (1999) Zbl 0952.35049 MR 1742215

[4] Almeida, L., Bethuel, F.: Topological methods for the Ginzburg-Landau equations. J. Math. Pures Appl. (9) 77, 1-49 (1998) Zbl 0904.35023 MR 1617594

[5] Andre, N., Bauman, P., Phillips, D.: Vortex pinning with bounded fields for the GinzburgLandau equation. Ann. Inst. H. Poincaré Anal. Non Linéaire 20, 705-729 (2003) Zbl 1040.35108 MR 1981405

[6] Berlyand, L., Mironescu, P.: Ginzburg-Landau minimizers with prescribed degrees: dependence on domain. C. R. Math. Acad. Sci. Paris 337, 375-380 (2003) Zbl 1035.49009 MR 2015079

[7] Berlyand, L., Mironescu, P.: Ginzburg-Landau minimizers with prescribed degrees. Capacity of the domain and emergence of vortices. Preprint, http://desargues.univ-lyon1.fr

[8] Berlyand, L., Mironescu, P.: Ginzburg-Landau minimizers with prescribed degrees. Capacity of the domain and emergence of vortices. J. Funct. Anal. 239, 76-99 (2006) Zbl 1131.58301 MR 2258217

[9] Berlyand, L., Golovaty, D., Rybalko, V.: Nonexistence of Ginzburg-Landau minimizers with prescribed degree on the boundary of a doubly connected domain. C. R. Math. Acad. Sci. Paris 343, 63-68 (2006) Zbl pre05046024 MR 2241961 
[10] Berlyand, L., Voss, K.: Symmetry breaking in annular domains for a Gizburg-Landau superconductivity model. In: IUTAM Symposium on Mechanical and Electromagnetic Waves in Structured Media (Sydney, 1999), Kluwer, 189-200 (2001)

[11] Bethuel, F., Brezis, H., Hélein, F.: Ginzburg-Landau Vortices. Birkhäuser (1994) Zbl 0802.35142 MR 1269538

[12] Boutet de Monvel-Berthier, A., Georgescu, V., Purice, R.: A boundary value problem related to the Ginzburg-Landau model. Comm. Math. Phys. 142, 1-23 (1991) Zbl 0742.35045 MR 1137773

[13] Brezis, H., Li, T. (eds.): Ginzburg-Landau Vortices. Ser. Contemp. Appl. Math. CAM 5, World Sci. (2005) Zbl 1089.35002

[14] Comte, M., Mironescu, P.: Minimizing properties of arbitrary solutions to the GinzburgLandau equation. Proc. Roy. Soc. Edinburgh Sect. A 129, 1157-1169(1999) Zbl 0941.35021 MR 1728535

[15] del Pino, M., Kowalczyk, M., Musso, M.: Variational reduction for Ginzburg-Landau vortices. J. Funct. Anal. 239, 497-541 (2006) Zbl pre05083435 MR 2261336

[16] Donnelly, R., Fetter, A.: Stability of superfluid flow in an annulus. Phys. Rev. Lett. 17, 747750 (1966)

[17] Elliott, C.-M., Matano, H., Tang, Q.: Zeros of a complex Ginzburg-Landau order parameter with applications to superconductivity. Eur. J. Appl. Math. 5, 431-448 (1994) Zbl 0817.35112 MR 1309733

[18] Fife, P.-C., Peletier, L.-A.: On the location of defects in stationary solutions of the Ginzburg-Landau equation in $\mathbb{R}^{2}$. Quart. Appl. Math. 54, 85-104 (1996) Zbl 0848.35042 MR 1373840

[19] Golovaty, D., Berlyand, L.: On uniqueness of vector-valued minimizers of the GinzburgLandau functional in annular domains. Calc. Var. Partial Differential Equations 14, 213-232 (2002) Zbl pre01756810 MR 1890400

[20] Jimbo, S., Morita, Y.: Stability of nonconstant steady-state solutions to a Ginzburg-Landau equation in higher space dimensions. Nonlinear Anal. 22, 753-770 (1994) Zbl 0798.35019 MR 1270168

[21] Jimbo, S., Morita, Y., Zhai, J.: Ginzburg-Landau equation and stable steady state solutions in a non-trivial domain. Comm. Partial Differential Equations 20, 2093-2112 (1995) Zbl 0841.35041 MR 1361731

[22] Jimbo, S., Sternberg, P.: Nonexistence of permanent currents in convex planar samples. SIAM J. Math. Anal. 33, 1379-1392 (2002) Zbl 1055.49031 MR 1920636

[23] Lin, F.-H.: Solutions of Ginzburg-Landau equations and critical points of the renormalized energy. Ann. Inst. H. Poincaré Anal. Non Linéaire 12, 599-622 (1995) Zbl 0845.35052 MR 1353261

[24] Lin, F.-H., Lin, T.-Ch.: Minimax solutions of the Ginzburg-Landau equations. Selecta Math. (N.S.) 3, 99-113 (1997) Zbl 0876.49006 MR 1454087

[25] Maz'ya, V. G.: Sobolev Spaces. Springer Ser. Soviet Math., Springer (1985) Zbl 0692.46023 MR 0817985

[26] Mironescu, P.: Explicit bounds for solutions to a Ginzburg-Landau type equation. Rev. Roumaine Math. Pures Appl. 41, 263-271 (1996) Zbl 0857.35021 MR 1423093

[27] Pacard, F., Rivière, T.: Linear and Nonlinear Aspects of Vortices. The Ginzburg-Landau Model. Progr. Nonlinear Differential Equations Appl. 39, Birkhäuser (2000) Zbl 0948.35003 MR 1763040

[28] Rubinstein, J., Sternberg, P.: Homotopy classification of minimizers of the Ginzburg-Landau energy and the existence of permanent currents. Comm. Math. Phys. 179, 257-263 (1996) Zbl 0860.35131 MR 1395224 
[29] Sandier, E., Serfaty, S.: Vortices in the Magnetic Ginzburg-Landau Model. Progr. Nonlinear Differential Equations Appl. 70, Birkhäuser (2007) Zbl 1112.35002 MR 2279839

[30] Serfaty, S.: Local minimizers for the Ginzburg-Landau energy near critical magnetic field, I, II. Comm. Contemp. Math. 1, 213-254, 295-333 (1999) Zbl 0944.49007, Zbl 0964.49005 MR 1707887, MR 1696100

[31] Serfaty, S.: Vortices in the Ginzburg-Landau model of superconductivity. In: Int. Congress of Mathematicians (Madrid, 2006), Vol. III, Eur. Math. Soc., Zurich, 267-290 (2006) Zbl 1130.82037 MR 2275681

[32] Serfaty, S.: Stability in 2D Ginzburg-Landau passes to the limit. Indiana Univ. Math. J. 54, 199-221 (2005) Zbl 1137.35074 MR 2126721

[33] Struwe, M.: On the asymptotic behavior of minimizers of the Ginzburg-Landau model in 2 dimensions. Differential Integral Equations 7, 1613-1624 (1994) Zbl 0809.35031 MR 1269674

[34] White, B.: Homotopy classes in Sobolev spaces and the existence of energy minimizing maps. Acta Math. 160, 1-17 (1988) Zbl 0647.58016 MR 0926523 